\title{
Química mineral de biotitas en neises de la Suite Metamórfica Rio Urubú aflorantes en la Serra Repartimento, Dominio Guiana Central en el Cratón Amazónico, Brasil: implicaciones petrogenéticas
}

\author{
Carlos Andrés Ballesteros-Camaro ${ }^{1 *}$; Johanna Ríos-Guerrero ${ }^{1}$
}

DOI: http://dx.doi.org/10.18273/revbol.v41n1-2019005 @ @ @

Forma de citar: Ballesteros-Camaro, C.A., y Ríos-Guerrero, J. (2019). Química mineral de biotitas en neises de la Suite Metamórfica Rio Urubú aflorantes en la Serra Repartimento, Dominio Guiana Central en el Cratón Amazónico, Brasil: implicaciones petrogenéticas. Boletín de Geología, 41(1), 97-116. DOI: 10.18273/revbol.v41n1-2019005.

\section{RESUMEN}

Ortoneises del basamento Paleoproteorozoico, de composición granítica de la Suite Metamórfica Rio Urubú (SMRU), relacionados a el evento pos-transamazónico son de amplia distribución en el Dominio Guiana Central (DGC) en el Cratón Amazónico, noreste de Brasil. En este estudio, fue estudiada la petrografía, mineralogía y química de biotitas en neises de biotitahornblenda pertenecientes a la SMRU. También fueron determinadas las condiciones de formación de estas biotitas y discutidas la importancia de estos minerales en términos petrogenéticos. Mediante los resultados de petrografía y de química mineral fueron clasificadas las biotitas en dos grupos distintos: 1) biotitas primarias clasificadas como Bt-IA, menos deformadas, de aspecto ígneo preservado y sin mayor orientación mineral y 2) biotitas primarias con tendencia a biotitas reequilibradas, con evidencia de deformación leve a moderada (Bt-IB), marcando la foliación de la roca y la estructura neísica, y siendo consistentes con los procesos tectono-metamórficos asociados a zonas de cizallamiento que afectaron el DGC. Los datos de microsonda electrónica muestran que los contenidos totales de Mg en biotitas menos deformadas (Bt-IA) son más altos, siendo clasificadas como biotitas magnesianas, mientras que las biotitas deformadas (Bt-IB) presentan contenidos mayores en Fe y siendo clasificadas como biotitas ferríreras. Resultados para biotitas estrictamente primarias indican que las rocas de la SMRU son asociadas a granitos provenientes de magmas tipo-I y afinidad geoquímica calco-alcalina, con temperaturas $(\mathrm{T})$ de cristalizaron para estas biotitas entre $720^{\circ} \mathrm{C}-760^{\circ} \mathrm{C}$, con condiciones de fugacidad de oxígeno $\left(f \mathrm{O}_{2}\right)$ de $-12,71$ y $-13,66$ y razones de $\mathrm{Fe} /(\mathrm{Fe}+\mathrm{Mg}) \geq 0,50$, mostrando una cristalización para estas rocas en el campo NNO, sugiriendo condiciones reductoras para el magma parental y también indicando una fuente típicamente de corteza para los magmas que dieron origen a esos granitos.

Palabras clave: Suite Metamórfica Rio Urubú; Química mineral; Biotita; Fugacidad de oxígeno; Magma parental.

Mineral chemistry of biotites in gneisses from the Rio Urubú Metamorphic Suite outcropping in the Serra Repartimento, Central Guyana Domain in the Amazonian Craton, Brazil: petrogenetic implications

\begin{abstract}
Orthogneisses of the Paleoproteorozoic basement from the Rio Urubú Metamorphic Suite (RUMS), related to the posttransamazonic event, are widely distributed in the Central Guyana Domain (CGD) in the Amazonian Craton, northeastern Brazil. In this study, it was investigated the petrography, mineralogy and chemistry of biotites within the biotite-hornblende gneisses from the RUMS. It was also determined the formation conditions of these biotites and discuss the significance of these minerals in terms of petrogenesis. It can be classified through the results of petrography and mineral chemistry biotites into two distinct groups: 1) primary biotite classified as Bt-IA, less deformed, igneous appearance preserved and with no mineral orientation; 2) primary biotite with a tendency to re-equilibrated biotite, classified as Bt-IB, with low to moderate evidence of deformation and showing the main foliation of the rock and the gneissic structure, and being consistent with the tectono-metamorphic processes associated with shear zones that affected the CGD. Microprobe data show that the total Mg contents in less deformed biotite (Bt-IA) are higher, classified as Mg-biotites, while the deformed biotite (Bt-IB) presents higher Fe content and are classified as Fe-biotites. Results for strictly primary biotite indicate that the rocks of the RUMS are associated to granites originated from an I-type magma and calc-alkaline geochemical affinity, where these biotites crystallized at temperatures $(\mathrm{T})$ de $720^{\circ} \mathrm{C}-760^{\circ} \mathrm{C}$, with oxygen fugacity $\left(f \mathrm{O}_{2}\right)$ conditions of -12.71 and $-13.66, \mathrm{Fe} /(\mathrm{Fe}+\mathrm{Mg}$ ) ratios $\geq 0.50$, showing crystallization for these rocks around the NNO buffer, suggesting reducing conditions for the parental magma and also indicating a typical crust source for the magmas that contributed to the formation of these granites.
\end{abstract}

Keywords: Rio Urubú Metamorphic Suite; Mineral chemistry; Biotite; oxygen fugacity; Parental magma.

1 Programa de Pós-graduação em Geociências, Universidade Federal do Amazonas, Manaus, Brasil. (*) carlosandres140@hotmail.com; joharios@gmail.com 


\section{INTRODUCCIÓN}

La biotita es el mineral ferromagnesiano significativo y más común en la mayoría de las rocas ígneas intermedias y félsicas, y una de las fases minerales más estudiadas como indicadores petrogenéticos y tectonomagmáticos en granitos (Lalonde y Bernard, 1993; Solie y Su, 1987; Speer, 1987; Masoudi y Jamshidi-Badr, 2008). En cuanto a la composición de la biotita, esta depende en gran medida de la naturaleza de los magmas de los que se han cristalizado (Abdel-Rahman, 1994; Shabani et al., 2003), y su potencial para reflejar la naturaleza y las condiciones físico-químicas de los magmas de los que se formó es alto (Masoudi y Jamshidi-Badr, 2008).

El área de estudio se encuentra localizada en el Escudo de las Guianas, al norte del Cratón Amazónico, geográficamente en la porción central del estado de Roraima, al suroeste de la cuidad de Boa Vista (FIGURA 1). En esta región, el plutonismo granítico asociado a una intensa trama de cizallamiento transcurrente es una de las características geológicas más marcadas en el ámbito del Dominio Guiana Central (DGC), presentando basamento de rocas ortoderivadas Paleoproterozóicas de alto grado metamórfico de la Suite Metamórfica Rio Urubú (SMRU), donde la ocurrencia de biotita es alta (10 - 11\%), y el estudio detallado de su mineralogía ha sido foco de pocos estudios. Con respecto al origen y geoquímica, muy pocos trabajos se han realizado para esta suite, determinándose que los magmas que formaron este cuerpo están asociados a magmas calco-alcalinos tipo I (Fraga, 2000), relacionados al magmatismo durante el pos-transamazónico (Fraga, 2000, 2002; Figueiredo, 2016).
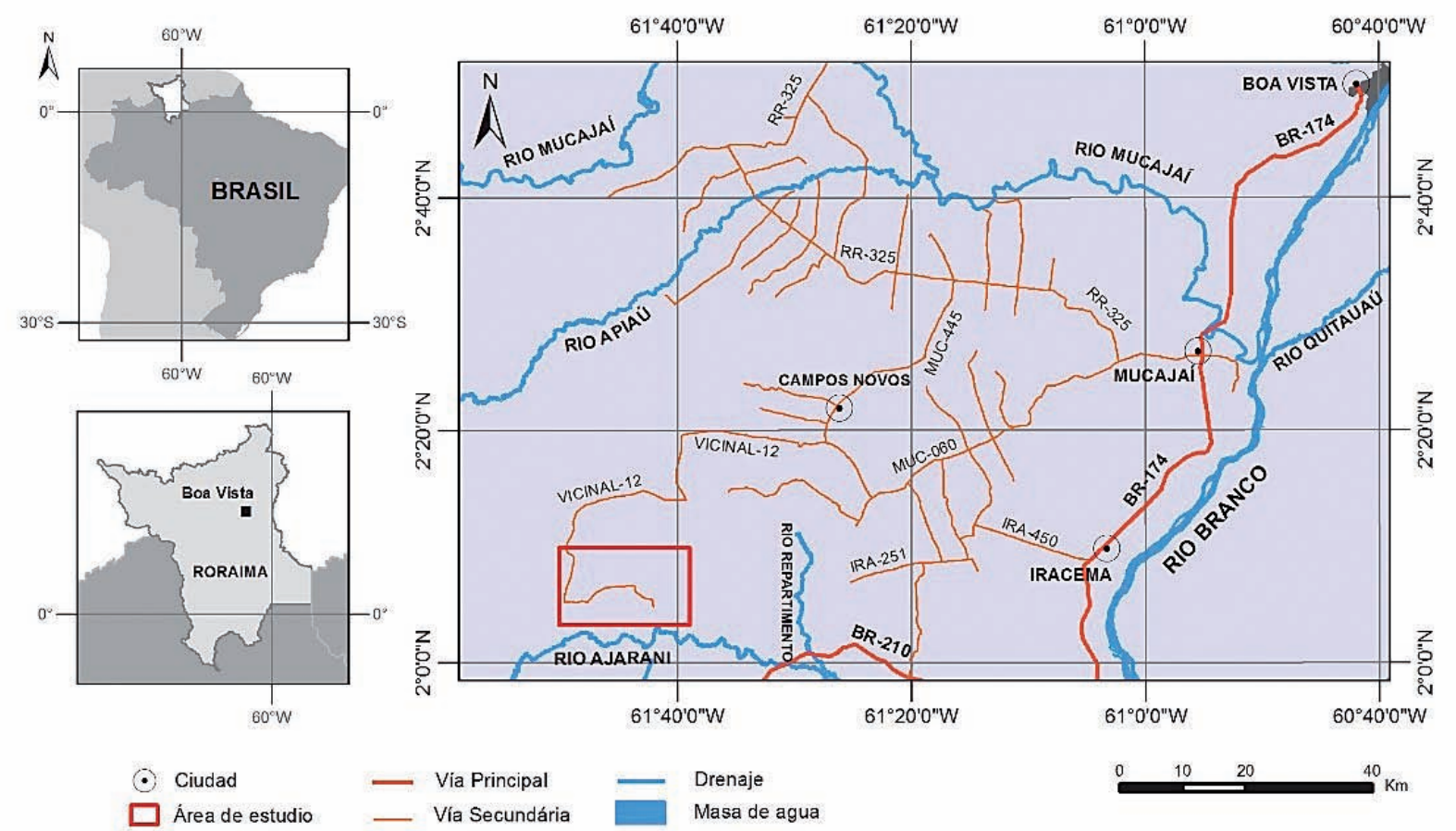

FIGURA 1. Mapa de localización del área de estudio con las principales vías de acceso y drenajes. En la izquierda mapas de Suramérica con destaque para Brasil y el estado de Roraima. En la derecha destaque de los municipios de Iracema, Mucajaí y Campos Novos con área de estudio de la Serra Repartimento en cuadro rojo.

En este trabajo son presentadas y discutidas la composición química de la biotita, principalmente primarias, de la SMRU mediante su estudio petrográfico, de química mineral y geotermometría, con el objetivo de investigar las características composicionales, petrogenéticas y aportar nuevas evidencias geoquímicas e inferir el origen del magma generador de esta suite.

\section{MARCO GEOLÓGICO}

El Cratón Amazónico (CA), situado en la porción norte de la plataforma Suramericana está constituido por los escudos de edad Arqueano-Proterozóicos, el de las Guianas en la parte norte, y escudo de BrasilCentral en la parte sur, geográficamente separados por las cuencas paleozóicas del Amazonas y Solimões 
(Almeida, 1978; Tassinari et al., 2000). Las litologías del CA engloban rocas metamórficas subdivididas en terrenos formados por complejos neísico-migmatíticos y granulíticos, suites granitoídes y terrenos de bajo grado metamórfico (Fraga, 1999, 2002; Tassinari y Macambira, 2004; Reis et al., 2004; Almeida, 2006; Rosa-Costa, 2006).

En este contexto, la región entorno de la Serra Repartimento, en el DGC, el cual se localiza en la porción centro-sur del estado de Roraima. Según el mapa geológico del estado de Roraima publicado por el Servicio Geológico Brasilero CPRM (1999) y modificado posteriormente por Fraga (2002; FIGURA 2A), ésta zona es constituida predominantemente por unidades litoestratigráficas de edades del Paleoproterozóico hasta el Mesozoico, representado por rocas del basamento Paleoproterozoico de ortoneises, metagranitoídes y cuerpos charnoquitícos de las Suites metamórfica Rio Urubú e Intrusiva Serra da Prata, además de intrusiones Mesoproterozoicas (granitoides, gabros y charnoquitos) de la Suite Intrusiva Mucajaí, Anortosito Repartimento y cuerpos alcalinos de edad Mesozoica del Complejo Alcalino Apiaú (Almeida et al., 2003). Este dominio es marcado por lineamientos fuertemente orientados en dirección NE-SW, donde por lo menos dos episodios Proterozoicos (pos-transamazónicos) proporcionaron su reactivación: a) uno más antiguo, de edad incierta, en facies anfibolita y b) otro en torno de 1,2 Ga (evento compresivo K'Mudku) en facies esquistos verdes (Santos et al., 2000).

La SMRU, objeto principal de este estudio, está compuesta por neises de composición granítica, inicialmente descritas por Fraga et al. (1994), representadas por neises biotíticos y biotíticohornbléndicos y metagranitoides, con subordinados lentes de cuarzo-mangeritas, cuarzo-jotunitos neísicos y leuconeises, mostrando una foliación definida en grado de metamorfismo en facies anfibolita (Fraga, 1999, 2002). Gaudette et al. (1996) obtuvieron por el método U-Pb en circón una edad de cristalización del protolito ígneo para los ortoneises de 1943 \pm 7

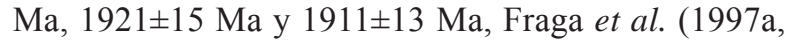
1997b) encontraron una edad de $1966 \pm 37$ Ma para una neis hipersténico, interpretado por Fraga (2000) como perteneciente a la SMRU. Posteriormente Fraga (2002) verificó una edad de $1937 \pm 5$ Ma y $1935 \pm 5$ Ma, por el método de $\mathrm{Pb}-\mathrm{Pb}$, para un neis hornbléndicobiotítico y un metagranito hornbléndico-biotítico, respectivamente. Recientemente, Figueiredo (2016) por el método de U-Pb en circón en rocas aflorantes en la región de Apiaú, en áreas cercanas a la Serra Repartimento, indicó una edad de $1931 \pm 4$ Ma e $1958 \pm 7$ Ma, confirmando la cristalización Paleoproterozoica para las rocas ortoderivadas de la SMRU, relacionadas al evento pos-transamazónico.

No obstante, en la región estudiada en la Serra Repartimento, afloran unidades ígneo-metamórficas de edad Paleoproterozoica, correspondientes a las SMRU y meta-gabros cartografiados por BallesterosCamaro (2017) y emplazados en los ortoneises (FIGURA 2B). En esta área las estructuras principales son zonas de cizallamiento con orientación preferencial NE-SW y subordinados E-W, siendo concordantes con la estructuración regional del DGC propuesto en la literatura.

\section{METODOLOGÍA Y TÉCNICAS ANALÍTICAS}

Para el propósito de este trabajo se recolectaron muestras de superficie representativas de neises correspondientes a la SMRU y se realizaron 10 secciones delgadas pulidas para su estudio mineralógico y petrográfico, las cuales se analizaron usando un microscopio marca Olympus, modelo BX51 del laboratorio de microscopia petrográfica de la Universidade Federal do Amazonas (UFAM).

A partir del estudio petrográfico fueron seleccionadas dos secciones delgadas pulidas representativas, y posteriormente metalizadas con carbono para análisis composicionales de biotitas mediante la microsonda electrónica, totalizando 34 puntos analizados. Los análisis de química mineral fueron efectuados en el Laboratorio de Microsonda Electrónica del Instituto de Geociencias de la Universidad de Brasilia, empleándose un equipo de fabricación JEOL JXA8230, con cinco espectrómetros WDS (WavelengthDispersive Spectroscopy) con cristales analizadores STE/TAP, TAP/PET, PET/LIF, PET/LIF, y PET/LF, $\mathrm{y}$ con detector de silicio EDS (Energy-Dispersive Spectroscopy). Las condiciones operacionales usadas durante las rutinas fueron con un potencial de activación de $15 \mathrm{kV}$ y corriente de haz de rayo electrónico de $20 \mathrm{nA}$, con diámetro de $5 \mu \mathrm{m}$ y tiempo de conteo de 20 segundos. El programa MICA ${ }^{+}$(Yavuz, 2003) se utilizó para los cálculos de las formulas estructurales mineralógicas de las micas, el cual considera el cálculo de $\mathrm{F}^{3+}$ con mayor confiabilidad. 

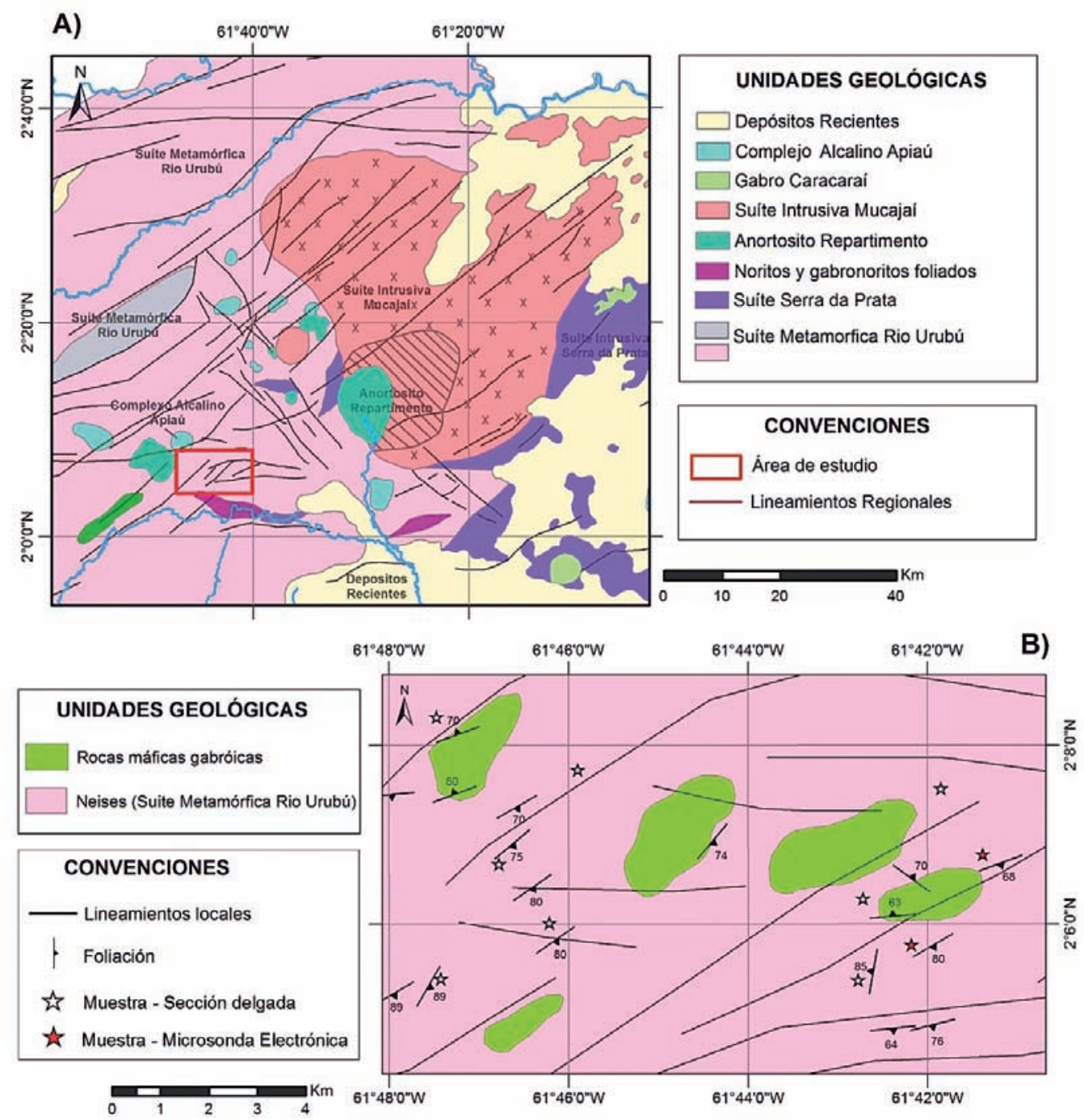

FIGURA 2. Litoestratigrafia del Domínio Guiana Central y Serra Repartimento. A. Mapa geológico regional con destaque del área de estudio, actualizado de Fraga (2002), Fraga et al. (2009a, 2009b), Costa et al. (2011), Heinonen et al. (2012), Tarazona (2015) y Figueiredo y Santos (2015). B. Mapa geológico de la Serra Repartimento producto del mapeamiento geológico realizado por Ballesteros-Camaro (2017).

\section{OCURRENCIA Y PETROGRAFÍA DE BIOTITAS}

En el área de la Serra Repartimento, la SMRU está comprendida por ortoneises de composición dominante granodiorítica a monzogranítica. Macroscópicamente, son rocas leucocráticas grisáceas claras a oscuras, con estructura bandeada y sectores donde la roca presenta aspecto ígneo preservado sin mayor orientación mineral. Está compuesta de porfiroclastos de feldespato alcalino con formas sigmoideas de hasta $3 \mathrm{~cm}$ de espesor y orientados principalmente NE/SE, siguiendo la estructura neísica y dando a la roca una textura tipo augen. La matriz es fina a media, cuarzo-feldespática con anfíbol, biotita y óxidos de Fe-Ti (FIGURA 3). Petrográficamente presentan una asociación mineral principal compuesta por feldespato alcalino + cuarzo + plagioclasa + biotita + hornblenda y, presentando como minerales accesorios titanita, epidota, alanita, minerales opacos (magnetita \pm ilmenita), apatita $\mathrm{y}$ circón (Ballesteros-Camaro, 2017).

En los neises con textura augen, la biotita es el mineral máfico principal y las microestructuras indican que la biotita es claramente primaria, sin embargo son observadas evidencias de transformaciones tardimagmáticas y/o alteraciones secundarias, tales como oxidación, más común, y cloritización menos común. 
En general, la biotita se presenta como cristales con habito laminar-placoso, dominantemente hipidomórficos, midiendo entre 0,1 y $0,25 \mathrm{~mm}$, con contactos rectos y cerrillados, de coloración amarilla verdosa, no obstante, en secciones basales muestran coloración marrón/castaño $\mathrm{y}$, comúnmente en contacto con cristales de cuarzo, feldespatos (alcalino y plagioclasa) y hornblenda junto a titanita. En algunas secciones la biotita define una leve a moderada orientación marcando la foliación de la roca y la estructura neísica, presentándose subidomórfica, con presencia de contactos irregulares y colores de pleocroísmo que varían de marrón claro a rojizo intenso (FIGURA 3). Inclusiones de minerales opacos, usualmente idiomórficos a subidomórficos de óxidos de Fe-Ti (magnetita-ilmenita), además de pequeños cristales idio-hipidomórficos de epidota, alanita y circón son comunes, generando halos pleocroicos. La cloritización ocurre esencialmente en bordas y en planos de clivaje de la biotita, llegando raramente a formar pseudomorfos. Las abreviaturas utilizadas en este trabajo son según Kretz (1983).

\section{QUÍMICA MINERAL}

Los análisis composicionales de las biotitas estudiadas están representadas en la TABLA 1, con la formula estructural, basada en 22 oxígenos equivalentes, con cierre analítico (sin tener en consideración el contenido de agua) $\geq 95 \%$, y sitios de ocupación llenados de acuerdo con Deer et al. (1997), donde la formula general es $\mathrm{X}_{2} \mathrm{Y}_{4-6} \mathrm{Z}_{8} \mathrm{O}_{20}(\mathrm{OH}, \mathrm{F})_{4}$ en que $\mathrm{X}$ $(\Sigma=2)$ es $\mathrm{K}^{1+}, \mathrm{Na}^{1+}, \mathrm{Ca}^{2+}, \mathrm{Ba}^{2+}, \mathrm{Rb}^{1+} \mathrm{y} \mathrm{Cs}^{1+}, \mathrm{Y}(\Sigma=6)$ es principalmente $\mathrm{Al}^{\mathrm{VI}}, \mathrm{Mg}^{2+}, \mathrm{Fe}^{2+}, \mathrm{Fe}^{3+}, \mathrm{Ti}^{4+}, \mathrm{Mn}^{3+}, \mathrm{Cr}^{3+}$, $\mathrm{Li}^{1+}$ y Z $(\Sigma=8)$ es $\mathrm{Si}^{4+}, \mathrm{Al}^{\mathrm{IV}}$ y Fe${ }^{3+}$. Estructuralmente, las micas se pueden clasificar como dioctaédricas $(\mathrm{Y}=4)$ y trioctaédricas $(\mathrm{Y}=6)$, siendo que las biotitas estudiadas demuestran una mica común trioctaédrica.

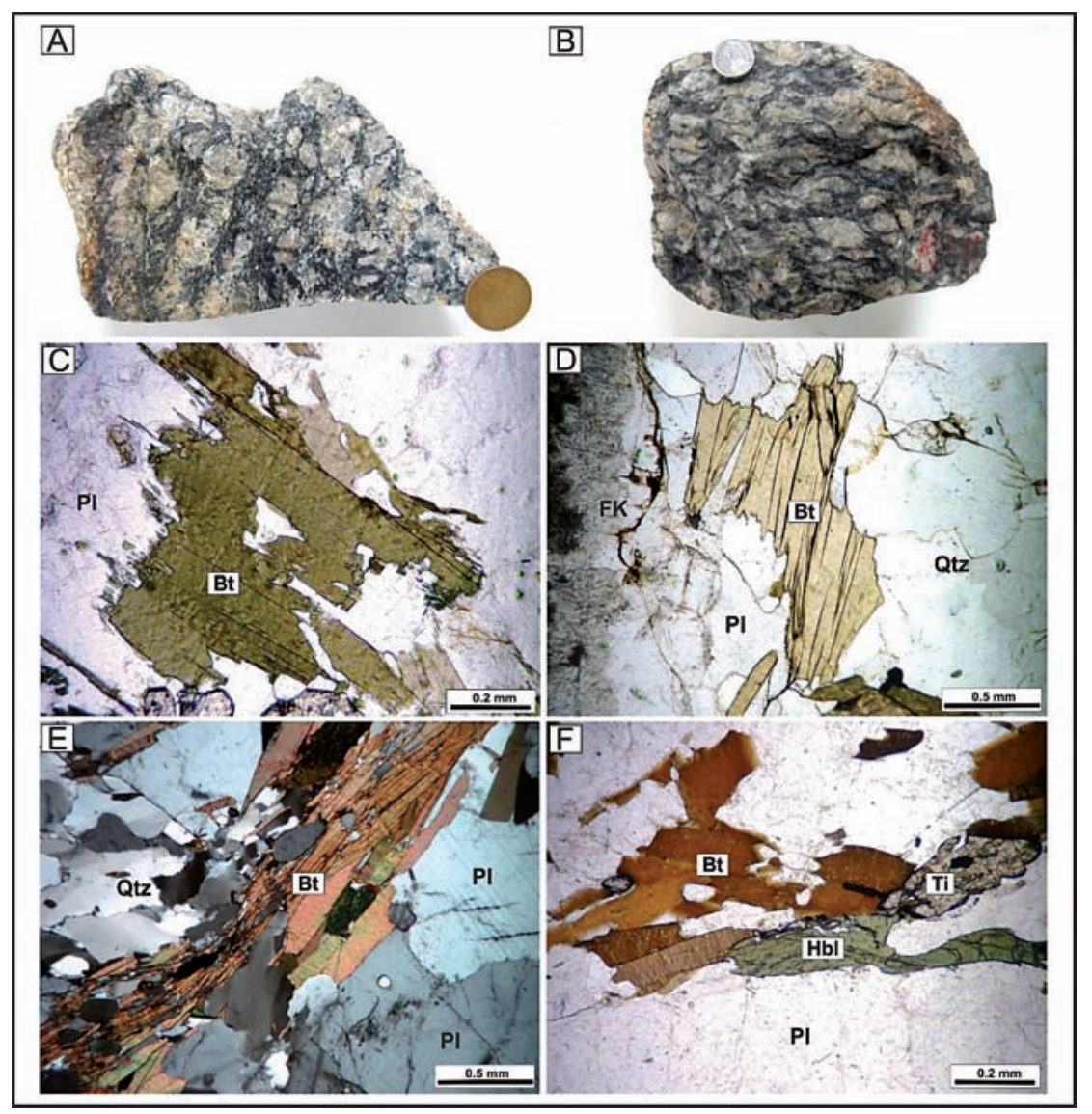

FIGURA 3. Aspectos macroscópicos y microscópicos de biotitas en neises con textura augen de la SMRU dependiendo del grado de afectación metamórfica de la roca. A. Aspecto macroscópico de las rocas del neis de la SMRU con textura ígnea preservada, sin mayor orientación mineral y estructura bandeada. B. Aspecto macroscópico de roca con estructura neísica con orientación mineral preferencial de augen de feldespato alcalino y cuarzo en matriz cuarzo-feldespática, biotita y hornblenda principalmente. C-D. Biotita hipidomórfica, laminar-placoso, de carácter ígneo preservado y sin mayor orientación en contacto con plagioclasa y cuarzo. E-F. Biotita subidomórfica en la forma de láminas orientadas en contacto con hornblenda y titanita marcando la foliación de la roca y la estructura neísica. Tamaño de la moneda en fotografía A: 2,0 cm de diámetro, en B: 1,5 cm. 
TABLA 1. Resultados de microsonda electrónica (wt\%) de biotitas en rocas neísicas de la SMRU. Los valores de Z, Y, X y A corresponden a las abreviaturas para cationes tetraédricos, octaédricos y aniones con base en 22 oxígenos. $\mathrm{X}_{\mathrm{ph}}, \mathrm{X}_{\mathrm{an}}, \mathrm{X}_{\mathrm{m}}, \mathrm{X}_{\mathrm{al}}$, $\mathrm{X}_{\mathrm{u}}=$ Fracciones molares de flogopita, anita, manganobiotita, aluminobiotita y titanobiotita según Jacobs y Parry (1979). El Índice de enriquecimiento de hierro (I.E) $=(\mathrm{Fe}+\mathrm{Mn}) / \mathrm{Fe}+\mathrm{Mn}+\mathrm{Mg})$. Numero de magnesio $(\mathrm{Mg} \#)=\mathrm{Mg} /(\mathrm{Mg}+\mathrm{Fe})$. Los cálculos correspondientes a miembros finales de micas (wt\%) de talco, Ti-flogopita, Fe-eastonita, moscovita y flogopita son tomados de Dymek (1983).

\begin{tabular}{|c|c|c|c|c|c|c|c|c|c|c|c|c|c|c|c|c|c|c|c|}
\hline \multirow{2}{*}{$\begin{array}{l}\text { PUNTO/ } \\
\text { WT\% }\end{array}$} & \multicolumn{19}{|c|}{ Biotitas (IA) - Muestra RM-14 } \\
\hline & 1_10 & 1_11 & 1_12 & 2_05 & 2_06 & 2_07 & 2_08 & 2_09 & 3_07 & 3_08 & 4_01 & 4_02 & 4_03 & 4_04 & 4_05 & 4_06 & 4_07 & 4_08 & 4_09 \\
\hline $\mathrm{SiO}_{2}$ & 35,72 & 36,40 & 35,97 & 35,75 & 36,01 & 35,73 & 35,80 & 36,13 & 36,06 & 35,68 & 36,16 & 36,18 & 35,61 & 35,98 & 36,01 & 36,01 & 36,06 & 35,29 & 35,92 \\
\hline $\mathrm{TiO}_{2}$ & 4,60 & 4,51 & 4,84 & 4,56 & 4,35 & 4,97 & 4,15 & 4,61 & 4,22 & 4,28 & 4,41 & 4,47 & 5,06 & 4,37 & 4,57 & 4,45 & 4,17 & 4,33 & 4,88 \\
\hline $\mathrm{Al}_{2} \mathrm{O}_{3}$ & 13,93 & 13,89 & 13,86 & 13,90 & 13,75 & 14,02 & 14,07 & 13,78 & 13,87 & 13,83 & 14,23 & 14,32 & 14,06 & 14,28 & 14,06 & 14,24 & 14,06 & 14,09 & 14,27 \\
\hline $\mathrm{Fe}_{2} \mathrm{O}_{3}$ & - & - & - & - & - & - & - & - & - & - & - & - & - & - & - & - & - & - & - \\
\hline $\mathrm{FeO}$ & 21,26 & 21,46 & 21,88 & 21,50 & 22,09 & 21,99 & 22,44 & 22,38 & 21,31 & 21,34 & 20,87 & 21,05 & 21,31 & 20,98 & 21,37 & 21,42 & 21,44 & 22,16 & 21,60 \\
\hline $\mathrm{MnO}$ & 0,27 & 0,13 & 0,31 & 0,35 & 0,28 & 0,31 & 0,31 & 0,30 & 0,39 & 0,21 & 0,24 & 0,31 & 0,42 & 0,25 & 0,30 & 0,41 & 0,27 & 0,21 & 0,40 \\
\hline MgO & 9,84 & 9,86 & 9,89 & 9,52 & 9,48 & 9,53 & 9,46 & 9,43 & 9,74 & 9,90 & 9,76 & 9,78 & 9,77 & 10,36 & 10,28 & 10,43 & 9,66 & 9,78 & 9,64 \\
\hline $\mathrm{CaO}$ & 0,01 & 0,00 & 0,01 & 0,01 & 0,03 & 0,04 & 0,03 & 0,03 & 0,02 & 0,02 & 0,01 & 0,00 & 0,00 & 0,02 & 0,00 & 0,00 & 0,00 & 0,00 & 0,00 \\
\hline $\mathrm{Na}_{2} \mathrm{O}$ & 0,11 & 0,08 & 0,09 & 0,06 & 0,04 & 0,05 & 0,06 & 0,09 & 0,12 & 0,11 & 0,11 & 0,06 & 0,12 & 0,14 & 0,06 & 0,06 & 0,14 & 0,15 & 0,16 \\
\hline $\mathrm{K}_{2} \mathrm{O}$ & 9,28 & 9,70 & 9,72 & 9,55 & 9,71 & 9,51 & 9,74 & 9,79 & 9,48 & 9,53 & 9,59 & 9,55 & 9,66 & 9,38 & 9,56 & 9,57 & 9,33 & 9,51 & 9,47 \\
\hline $\mathrm{F}$ & 0,47 & 0,54 & 0,50 & 0,34 & 0,43 & 0,53 & 0,52 & 0,43 & 0,51 & 0,60 & 0,53 & 0,52 & 0,35 & 0,48 & 0,55 & 0,70 & 0,47 & 0,52 & 0,45 \\
\hline $\mathrm{Cl}$ & 0,11 & 0,10 & 0,11 & 0,07 & 0,13 & 0,09 & 0,09 & 0,07 & 0,09 & 0,09 & 0,10 & 0,10 & 0,10 & 0,08 & 0,08 & 0,09 & 0,07 & 0,12 & 0,09 \\
\hline $\mathrm{Cr}_{2} \mathrm{O}_{3}$ & 0,11 & 0,09 & 0,03 & 0,06 & 0,03 & 0,00 & 0,05 & 0,02 & 0,00 & 0,10 & 0,10 & 0,00 & 0,07 & 0,00 & 0,00 & 0,02 & 0,04 & 0,02 & 0,07 \\
\hline $\mathrm{V}_{2} \mathrm{O}_{3}$ & 0,08 & 0,01 & 0,00 & 0,08 & 0,10 & 0,04 & 0,07 & 0,01 & 0,03 & 0,01 & 0,04 & 0,09 & 0,06 & 0,12 & 0,04 & 0,01 & 0,08 & 0,12 & 0,10 \\
\hline $\mathrm{NiO}$ & 0,00 & 0,00 & 0,01 & 0,05 & 0,00 & 0,03 & 0,05 & 0,06 & 0,00 & 0,04 & 0,04 & 0,06 & 0,00 & 0,08 & 0,01 & 0,00 & 0,02 & 0,01 & 0,00 \\
\hline $\mathrm{Li}_{2} \mathrm{O}(\mathrm{c})$ & 0,70 & 0,89 & 0,77 & 0,71 & 0,78 & 0,70 & 0,72 & 0,82 & 0,80 & 0,69 & 0,82 & 0,83 & 0,67 & 0,78 & 0,78 & 0,78 & 0,80 & 0,58 & 0,76 \\
\hline Total & 96,50 & 97,65 & 97,98 & 96,48 & 97,21 & 97,52 & 97,55 & 97,93 & 96,63 & 96,42 & 97,02 & 97,31 & 97,23 & 97,31 & 97,67 & 98,20 & 96,60 & 96,87 & 97,79 \\
\hline \multicolumn{20}{|c|}{ Formula Mineral 22 Oxígenos } \\
\hline $\mathrm{Si}$ & 5,54 & 5,59 & 5,52 & 5,55 & 5,58 & 5,51 & 5,54 & 5,56 & 5,59 & 5,55 & 5,57 & 5,57 & 5,49 & 5,53 & 5,53 & 5,51 & 5,59 & 5,48 & 5,51 \\
\hline $\mathrm{Al}(\mathrm{IV})$ & 2,46 & 2,41 & 2,48 & 2,45 & 2,42 & 2,49 & 2,46 & 2,44 & 2,41 & 2,45 & 2,43 & 2,43 & 2,51 & 2,47 & 2,47 & 2,49 & 2,41 & 2,52 & 2,49 \\
\hline $\mathrm{Fe}^{+3}$ & 0,00 & 0,00 & 0,00 & 0,00 & 0,00 & 0,00 & 0,00 & 0,00 & 0,00 & 0,00 & 0,00 & 0,00 & 0,00 & 0,00 & 0,00 & 0,00 & 0,00 & 0,00 & 0,00 \\
\hline Suma (Z) & 8,00 & 8,00 & 8,00 & 8,00 & 8,00 & 8,00 & 8,00 & 8,00 & 8,00 & 8,00 & 8,00 & 8,00 & 8,00 & 8,00 & 8,00 & 8,00 & 8,00 & 8,00 & 8,00 \\
\hline $\mathrm{Al}(\mathrm{VI})$ & 0,09 & 0,10 & 0,03 & 0,10 & 0,09 & 0,05 & 0,10 & 0,06 & 0,13 & 0,09 & 0,16 & 0,16 & 0,04 & 0,12 & 0,07 & 0,07 & 0,16 & 0,07 & 0,09 \\
\hline $\mathrm{Cr}$ & 0,02 & 0,02 & 0,00 & 0,01 & 0,00 & 0,00 & 0,01 & 0,00 & 0,00 & 0,01 & 0,01 & 0,00 & 0,01 & 0,00 & 0,00 & 0,00 & 0,01 & 0,00 & 0,01 \\
\hline $\mathrm{Ti}$ & 0,54 & 0,52 & 0,56 & 0,53 & 0,51 & 0,58 & 0,48 & 0,53 & 0,49 & 0,50 & 0,51 & 0,52 & 0,59 & 0,51 & 0,53 & 0,51 & 0,49 & 0,51 & 0,56 \\
\hline $\mathrm{Fe}^{+3}$ & 0,73 & 0,56 & 0,78 & 0,64 & 0,58 & 0,81 & 0,60 & 0,63 & 0,54 & 0,62 & 0,49 & 0,57 & 0,80 & 0,69 & 0,77 & 0,80 & 0,54 & 0,76 & 0,74 \\
\hline $\mathrm{Fe}^{+2}$ & 2,03 & 2,20 & 2,03 & 2,15 & 2,28 & 2,02 & 2,30 & 2,25 & 2,22 & 2,16 & 2,20 & 2,14 & 1,95 & 2,00 & 1,97 & 1,94 & 2,24 & 2,12 & 2,03 \\
\hline $\mathrm{Mn}$ & 0,04 & 0,02 & 0,04 & 0,05 & 0,04 & 0,04 & 0,04 & 0,04 & 0,05 & 0,03 & 0,03 & 0,04 & 0,05 & 0,03 & 0,04 & 0,05 & 0,04 & 0,03 & 0,05 \\
\hline $\mathrm{Mg}$ & 2,27 & 2,26 & 2,26 & 2,20 & 2,19 & 2,19 & 2,18 & 2,16 & 2,25 & 2,30 & 2,24 & 2,24 & 2,24 & 2,37 & 2,35 & 2,38 & 2,23 & 2,27 & 2,20 \\
\hline $\mathrm{Li}$ & 0,21 & 0,27 & 0,24 & 0,22 & 0,24 & 0,22 & 0,23 & 0,26 & 0,25 & 0,21 & 0,26 & 0,26 & 0,21 & 0,24 & 0,24 & 0,24 & 0,25 & 0,18 & 0,23 \\
\hline Suma (Y) & 5,92 & 5,94 & 5,94 & 5,90 & 5,93 & 5,91 & 5,94 & 5,93 & 5,94 & 5,92 & 5,91 & 5,93 & 5,89 & 5,97 & 5,97 & 6,00 & 5,94 & 5,93 & 5,92 \\
\hline $\mathrm{Ca}$ & 0,00 & 0,00 & 0,00 & 0,00 & 0,00 & 0,01 & 0,00 & 0,01 & 0,00 & 0,00 & 0,00 & 0,00 & 0,00 & 0,00 & 0,00 & 0,00 & 0,00 & 0,00 & 0,00 \\
\hline $\mathrm{Na}$ & 0,03 & 0,02 & 0,03 & 0,02 & 0,01 & 0,01 & 0,02 & 0,03 & 0,04 & 0,03 & 0,03 & 0,02 & 0,04 & 0,04 & 0,02 & 0,02 & 0,04 & 0,04 & 0,05 \\
\hline K & 1,84 & 1,90 & 1,90 & 1,89 & 1,92 & 1,87 & 1,92 & 1,92 & 1,88 & 1,89 & 1,89 & 1,87 & 1,90 & 1,84 & 1,87 & 1,87 & 1,85 & 1,89 & 1,85 \\
\hline Suma $(X)$ & 1,87 & 1,92 & 1,93 & 1,91 & 1,94 & 1,89 & 1,95 & 1,95 & 1,92 & 1,93 & 1,92 & 1,89 & 1,94 & 1,89 & 1,89 & 1,88 & 1,89 & 1,94 & 1,90 \\
\hline $\mathrm{OH}$ & 3,74 & 3,71 & 3,73 & 3,82 & 3,75 & 3,72 & 3,72 & 3,78 & 3,72 & 3,68 & 3,72 & 3,72 & 3,81 & 3,74 & 3,71 & 3,64 & 3,75 & 3,71 & 3,76 \\
\hline $\mathrm{F}$ & 0,23 & 0,26 & 0,24 & 0,17 & 0,21 & 0,26 & 0,26 & 0,21 & 0,25 & 0,29 & 0,26 & 0,25 & 0,17 & 0,23 & 0,27 & 0,34 & 0,23 & 0,26 & 0,22 \\
\hline $\mathrm{Cl}$ & 0,03 & 0,03 & 0,03 & 0,02 & 0,03 & 0,02 & 0,02 & 0,02 & 0,02 & 0,02 & 0,03 & 0,03 & 0,03 & 0,02 & 0,02 & 0,02 & 0,02 & 0,03 & 0,02 \\
\hline Suma (A) & 4,00 & 4,00 & 4,00 & 4,00 & 4,00 & 4,00 & 4,00 & 4,00 & 4,00 & 4,00 & 4,00 & 4,00 & 4,00 & 4,00 & 4,00 & 4,00 & 4,00 & 4,00 & 4,00 \\
\hline Xph & 0,38 & 0,38 & 0,38 & 0,37 & 0,37 & 0,37 & 0,37 & 0,37 & 0,38 & 0,39 & 0,38 & 0,38 & 0,38 & 0,40 & 0,39 & 0,40 & 0,38 & 0,38 & 0,37 \\
\hline Xan & 0,34 & 0,37 & 0,34 & 0,37 & 0,39 & 0,34 & 0,39 & 0,38 & 0,38 & 0,37 & 0,37 & 0,36 & 0,33 & 0,34 & 0,33 & 0,32 & 0,38 & 0,36 & 0,34 \\
\hline $\mathrm{Xmn}$ & 0,01 & 0,00 & 0,01 & 0,01 & 0,01 & 0,01 & 0,01 & 0,01 & 0,01 & 0,01 & 0,01 & 0,01 & 0,01 & 0,01 & 0,01 & 0,01 & 0,01 & 0,01 & 0,01 \\
\hline Xal & 0,02 & 0,02 & 0,01 & 0,02 & 0,02 & 0,01 & 0,02 & 0,01 & 0,02 & 0,02 & 0,03 & 0,03 & 0,01 & 0,02 & 0,01 & 0,01 & 0,03 & 0,01 & 0,02 \\
\hline Xti & 0,09 & 0,09 & 0,09 & 0,09 & 0,09 & 0,10 & 0,08 & 0,09 & 0,08 & 0,09 & 0,09 & 0,09 & 0,10 & 0,09 & 0,09 & 0,09 & 0,08 & 0,09 & 0,10 \\
\hline
\end{tabular}




\begin{tabular}{|c|c|c|c|c|c|c|c|c|c|c|c|c|c|c|c|c|c|c|c|}
\hline \multirow{2}{*}{$\begin{array}{c}\text { PUNTO/ } \\
\text { WT\% }\end{array}$} & \multicolumn{19}{|c|}{ Biotitas (IA) - Muestra RM-14 } \\
\hline & 1_10 & 1_11 & 1_12 & 2_05 & 2_06 & 2_07 & 2_08 & 2_09 & 3_07 & 3_08 & 4_01 & 4_02 & 4_03 & 4_04 & 4_05 & 4_06 & 4_07 & 4_08 & 4_09 \\
\hline I.E & 0,55 & 0,55 & 0,56 & 0,56 & 0,57 & 0,57 & 0,57 & 0,57 & 0,56 & 0,55 & 0,55 & 0,55 & 0,56 & 0,54 & 0,54 & 0,54 & 0,56 & 0,56 & 0,56 \\
\hline $\mathrm{Mg \#}$ & 0,45 & 0,45 & 0,45 & 0,44 & 0,43 & 0,44 & 0,43 & 0,43 & 0,45 & 0,45 & 0,46 & 0,45 & 0,45 & 0,47 & 0,46 & 0,47 & 0,45 & 0,44 & 0,44 \\
\hline $\mathrm{Fe} / \mathrm{Fe}+\mathrm{Mg}$ & 0,55 & 0,55 & 0,55 & 0,56 & 0,57 & 0,56 & 0,57 & 0,57 & 0,55 & 0,55 & 0,55 & 0,55 & 0,55 & 0,53 & 0,54 & 0,54 & 0,56 & 0,56 & 0,56 \\
\hline $\mathrm{Mg} / \mathrm{Mg}+\mathrm{Fe}$ & 0,45 & 0,45 & 0,45 & 0,44 & 0,43 & 0,44 & 0,43 & 0,43 & 0,45 & 0,45 & 0,45 & 0,45 & 0,45 & 0,47 & 0,46 & 0,46 & 0,45 & 0,44 & 0,44 \\
\hline $\mathrm{Fe}^{3} / \mathrm{Fe}^{2}+\mathrm{Fe}^{3}$ & 0,26 & 0,20 & 0,28 & 0,23 & 0,20 & 0,29 & 0,21 & 0,22 & 0,20 & 0,22 & 0,18 & 0,21 & 0,29 & 0,26 & 0,28 & 0,29 & 0,20 & 0,27 & 0,27 \\
\hline $\mathrm{Fe}^{2} / \mathrm{Mg}+\mathrm{Fe}^{2}$ & 0,47 & 0,49 & 0,47 & 0,49 & 0,51 & 0,48 & 0,51 & 0,51 & 0,50 & 0,49 & 0,50 & 0,49 & 0,47 & 0,46 & 0,46 & 0,45 & 0,50 & 0,48 & 0,48 \\
\hline Flogopita & 24,06 & 37,33 & 23,18 & 31,61 & 37,74 & 18,37 & 37,91 & 34,51 & 39,64 & 35,38 & 41,45 & 35,67 & 21,02 & 28,49 & 23,04 & 21,65 & 38,28 & 27,01 & 23,76 \\
\hline Ti-Flogopita & 26,85 & 26,05 & 27,94 & 26,62 & 25,32 & 28,80 & 24,13 & 26,65 & 24,59 & 25,03 & 25,59 & 25,86 & 29,32 & 25,27 & 26,37 & 25,60 & 24,28 & 25,32 & 28,12 \\
\hline Ferro-easto & 36,48 & 28,02 & 38,90 & 32,03 & 28,96 & 40,59 & 30,17 & 31,34 & 27,01 & 30,89 & 24,53 & 28,40 & 39,78 & 34,73 & 38,62 & 40,21 & 27,21 & 38,20 & 37,00 \\
\hline Eastonita & 0,00 & 0,00 & 0,00 & 0,00 & 0,00 & 0,00 & 0,00 & 0,00 & 0,00 & 0,00 & 0,49 & 0,00 & 0,00 & 0,00 & 0,00 & 0,00 & 0,00 & 0,00 & 0,00 \\
\hline Talco & 6,53 & 3,94 & 3,51 & 4,40 & 3,15 & 5,48 & 2,76 & 2,28 & 4,25 & 3,54 & 3,86 & 5,35 & 3,25 & 5,73 & 5,53 & 5,84 & 5,70 & 3,10 & 4,95 \\
\hline
\end{tabular}

\section{Continuación TABLA 1}

\begin{tabular}{|c|c|c|c|c|c|c|c|c|c|c|c|c|c|c|c|}
\hline \multirow{2}{*}{ PUNTO/WT\% } & \multicolumn{15}{|c|}{ Biotitas (IB) - Muestra RM-007 } \\
\hline & 1_08 & 1_09 & 1_11 & 1_12 & 2_05 & 2_06 & 3_01 & 3_02 & 3_03 & 3_04 & 3_05 & 3_06 & 4_01 & 4_02 & 4_03 \\
\hline $\mathrm{SiO}_{2}$ & 35,34 & 35,12 & 35,36 & 34,92 & 35,31 & 34,91 & 35,07 & 35,12 & 34,86 & 35,17 & 34,96 & 35,23 & 35,01 & 35,38 & 35,29 \\
\hline $\mathrm{TiO}_{2}$ & 3,47 & 3,35 & 3,28 & 3,01 & 3,91 & 3,94 & 3,49 & 3,37 & 3,72 & 3,60 & 2,87 & 3,03 & 3,78 & 3,08 & 3,41 \\
\hline $\mathrm{Al}_{2} \mathrm{O}_{3}$ & 14,54 & 15,14 & 15,32 & 15,48 & 15,05 & 14,98 & 15,27 & 15,06 & 15,12 & 15,23 & 14,91 & 14,58 & 14,92 & 15,10 & 14,94 \\
\hline $\mathrm{Fe}_{2} \mathrm{O}_{3}$ & - & - & - & - & - & - & - & - & - & - & - & - & - & - & - \\
\hline $\mathrm{FeO}$ & 24,19 & 24,79 & 24,50 & 23,99 & 24,00 & 23,57 & 24,79 & 23,99 & 24,23 & 24,83 & 24,62 & 25,36 & 24,53 & 24,06 & 24,85 \\
\hline $\mathrm{MnO}$ & 0,49 & 0,50 & 0,38 & 0,35 & 0,51 & 0,52 & 0,42 & 0,56 & 0,35 & 0,44 & 0,49 & 0,53 & 0,69 & 0,53 & 0,37 \\
\hline MgO & 6,86 & 7,05 & 6,84 & 7,31 & 6,31 & 6,49 & 7,01 & 7,00 & 7,17 & 6,97 & 6,83 & 7,00 & 6,63 & 6,95 & 7,12 \\
\hline $\mathrm{CaO}$ & 0,02 & 0,00 & 0,04 & 0,04 & 0,02 & 0,00 & 0,01 & 0,01 & 0,00 & 0,00 & 0,04 & 0,00 & 0,00 & 0,01 & 0,00 \\
\hline $\mathrm{Na}_{2} \mathrm{O}$ & 0,17 & 0,00 & 0,07 & 0,03 & 0,06 & 0,11 & 0,05 & 0,06 & 0,02 & 0,09 & 0,17 & 0,12 & 0,07 & 0,10 & 0,05 \\
\hline $\mathrm{K}_{2} \mathrm{O}$ & 9,59 & 9,69 & 9,43 & 9,52 & 9,74 & 9,48 & 9,46 & 9,51 & 9,82 & 9,51 & 9,43 & 9,50 & 9,65 & 9,32 & 9,44 \\
\hline $\mathrm{F}$ & 0,42 & 0,34 & 0,64 & 0,37 & 0,68 & 0,71 & 0,54 & 0,67 & 0,28 & 0,52 & 0,40 & 0,45 & 0,79 & 0,68 & 0,72 \\
\hline $\mathrm{Cl}$ & 0,15 & 0,09 & 0,09 & 0,08 & 0,10 & 0,09 & 0,07 & 0,09 & 0,04 & 0,10 & 0,10 & 0,07 & 0,08 & 0,10 & 0,10 \\
\hline $\mathrm{Cr}_{2} \mathrm{O}_{3}$ & 0,00 & 0,00 & 0,00 & 0,00 & 0,00 & 0,09 & 0,02 & 0,08 & 0,11 & 0,09 & 0,00 & 0,08 & 0,03 & 0,01 & 0,05 \\
\hline $\mathrm{V}_{2} \mathrm{O}_{3}$ & 0,04 & 0,03 & 0,03 & 0,07 & 0,09 & 0,08 & 0,06 & 0,10 & 0,05 & 0,06 & 0,12 & 0,08 & 0,04 & 0,08 & 0,11 \\
\hline $\mathrm{NiO}$ & 0,00 & 0,06 & 0,13 & 0,02 & 0,04 & 0,06 & 0,04 & 0,03 & 0,04 & 0,00 & 0,07 & 0,06 & 0,01 & 0,03 & 0,00 \\
\hline $\mathrm{Li}_{2} \mathrm{O}(\mathrm{c})$ & 0,59 & 0,53 & 0,60 & 0,47 & 0,58 & 0,47 & 0,51 & 0,53 & 0,45 & 0,54 & 0,48 & 0,56 & 0,50 & 0,60 & 0,58 \\
\hline Total & 95,86 & 96,70 & 96,69 & 95,66 & 96,40 & 95,48 & 96,81 & 96,16 & 96,26 & 97,14 & 95,48 & 96,64 & 96,72 & 96,04 & 97,03 \\
\hline \multicolumn{16}{|c|}{ Formula Mineral 22 Oxígenos } \\
\hline $\mathrm{Si}$ & 5,60 & 5,52 & 5,56 & 5,52 & 5,58 & 5,56 & 5,51 & 5,56 & 5,49 & 5,51 & 5,57 & 5,57 & 5,53 & 5,60 & 5,55 \\
\hline $\mathrm{Al}(\mathrm{IV})$ & 2,40 & 2,48 & 2,44 & 2,48 & 2,42 & 2,44 & 2,49 & 2,44 & 2,51 & 2,49 & 2,43 & 2,43 & 2,47 & 2,40 & 2,45 \\
\hline $\mathrm{Fe}^{+3}$ & 0,00 & 0,00 & 0,00 & 0,00 & 0,00 & 0,00 & 0,00 & 0,00 & 0,00 & 0,00 & 0,00 & 0,00 & 0,00 & 0,00 & 0,00 \\
\hline Suma $(Z)$ & 8,00 & 8,00 & 8,00 & 8,00 & 8,00 & 8,00 & 8,00 & 8,00 & 8,00 & 8,00 & 8,00 & 8,00 & 8,00 & 8,00 & 8,00 \\
\hline $\mathrm{Al}(\mathrm{VI})$ & 0,32 & 0,33 & 0,40 & 0,41 & 0,38 & 0,37 & 0,34 & 0,37 & 0,30 & 0,33 & 0,38 & 0,29 & 0,31 & 0,41 & 0,32 \\
\hline $\mathrm{Cr}$ & 0,00 & 0,00 & 0,00 & 0,00 & 0,00 & 0,02 & 0,00 & 0,01 & 0,01 & 0,01 & 0,00 & 0,01 & 0,00 & 0,00 & 0,01 \\
\hline $\mathrm{Ti}$ & 0,41 & 0,40 & 0,39 & 0,36 & 0,46 & 0,47 & 0,41 & 0,40 & 0,44 & 0,42 & 0,34 & 0,36 & 0,45 & 0,37 & 0,40 \\
\hline $\mathrm{Fe}^{+3}$ & 0,12 & 0,31 & 0,17 & 0,20 & 0,09 & 0,18 & 0,35 & 0,21 & 0,33 & 0,35 & 0,10 & 0,27 & 0,30 & 0,12 & 0,34 \\
\hline $\mathrm{Fe}^{+2}$ & 3,09 & 2,95 & 3,06 & 2,98 & 3,08 & 2,96 & 2,90 & 2,97 & 2,86 & 2,91 & 3,18 & 3,09 & 2,94 & 3,07 & 2,93 \\
\hline $\mathrm{Mn}$ & 0,07 & 0,07 & 0,05 & 0,05 & 0,07 & 0,07 & 0,06 & 0,07 & 0,05 & 0,06 & 0,07 & 0,07 & 0,09 & 0,07 & 0,05 \\
\hline $\mathrm{Mg}$ & 1,62 & 1,65 & 1,60 & 1,72 & 1,48 & 1,54 & 1,64 & 1,65 & 1,68 & 1,63 & 1,62 & 1,65 & 1,56 & 1,64 & 1,67 \\
\hline $\mathrm{Li}$ & 0,19 & 0,17 & 0,19 & 0,15 & 0,19 & 0,13 & 0,16 & 0,16 & 0,13 & 0,16 & 0,15 & 0,17 & 0,15 & 0,19 & 0,17 \\
\hline
\end{tabular}




\begin{tabular}{|c|c|c|c|c|c|c|c|c|c|c|c|c|c|c|c|}
\hline \multirow{2}{*}{ PUNTO/WT\% } & \multicolumn{15}{|c|}{ Biotitas (IB) - Muestra RM-007 } \\
\hline & 1_08 & 1_09 & 1_11 & 1_12 & 2_05 & 2_06 & 3_01 & 3_02 & 3_03 & 3_04 & 3_05 & 3_06 & 4_01 & 4_02 & 4_03 \\
\hline Suma $(\mathbf{Y})$ & 5,81 & 5,87 & 5,86 & 5,86 & 5,75 & 5,74 & 5,87 & 5,84 & 5,81 & 5,86 & 5,84 & 5,91 & 5,81 & 5,86 & 5,89 \\
\hline $\mathrm{Ca}$ & 0,00 & 0,00 & 0,01 & 0,01 & 0,00 & 0,00 & 0,00 & 0,00 & 0,00 & 0,00 & 0,01 & 0,00 & 0,00 & 0,00 & 0,00 \\
\hline $\mathrm{Na}$ & 0,05 & 0,00 & 0,02 & 0,01 & 0,02 & 0,03 & 0,02 & 0,02 & 0,01 & 0,03 & 0,05 & 0,04 & 0,02 & 0,03 & 0,02 \\
\hline K & 1,94 & 1,94 & 1,89 & 1,92 & 1,96 & 1,92 & 1,90 & 1,92 & 1,97 & 1,90 & 1,92 & 1,92 & 1,94 & 1,88 & 1,89 \\
\hline Suma $(\mathbf{X})$ & 2,00 & 1,94 & 1,92 & 1,94 & 1,98 & 1,96 & 1,91 & 1,94 & 1,98 & 1,93 & 1,98 & 1,95 & 1,97 & 1,91 & 1,91 \\
\hline $\mathrm{OH}$ & 3,75 & 3,81 & 3,66 & 3,79 & 3,63 & 3,62 & 3,71 & 3,64 & 3,85 & 3,72 & 3,77 & 3,76 & 3,58 & 3,63 & 3,62 \\
\hline $\mathrm{F}$ & 0,21 & 0,17 & 0,32 & 0,18 & 0,34 & 0,36 & 0,27 & 0,34 & 0,14 & 0,26 & 0,20 & 0,22 & 0,39 & 0,34 & 0,36 \\
\hline $\mathrm{Cl}$ & 0,04 & 0,02 & 0,02 & 0,02 & 0,03 & 0,02 & 0,02 & 0,02 & 0,01 & 0,03 & 0,03 & 0,02 & 0,02 & 0,03 & 0,03 \\
\hline Suma (A) & 4,00 & 4,00 & 4,00 & 4,00 & 4,00 & 4,00 & 4,00 & 4,00 & 4,00 & 4,00 & 4,00 & 4,00 & 4,00 & 4,00 & 4,00 \\
\hline Xph & 0,28 & 0,28 & 0,27 & 0,29 & 0,26 & 0,27 & 0,28 & 0,28 & 0,29 & 0,28 & 0,28 & 0,28 & 0,27 & 0,28 & 0,28 \\
\hline Xan & 0,53 & 0,50 & 0,52 & 0,51 & 0,54 & 0,52 & 0,50 & 0,51 & 0,49 & 0,50 & 0,54 & 0,52 & 0,51 & 0,52 & 0,50 \\
\hline $\mathrm{Xmn}$ & 0,01 & 0,01 & 0,01 & 0,01 & 0,01 & 0,01 & 0,01 & 0,01 & 0,01 & 0,01 & 0,01 & 0,01 & 0,02 & 0,01 & 0,01 \\
\hline Xal & 0,06 & 0,06 & 0,07 & 0,07 & 0,07 & 0,06 & 0,06 & 0,06 & 0,05 & 0,06 & 0,06 & 0,05 & 0,05 & 0,07 & 0,05 \\
\hline Xti & 0,07 & 0,07 & 0,07 & 0,06 & 0,08 & 0,08 & 0,07 & 0,07 & 0,08 & 0,07 & 0,06 & 0,06 & 0,08 & 0,06 & 0,07 \\
\hline I.E & 0,67 & 0,67 & 0,67 & 0,65 & 0,69 & 0,68 & 0,67 & 0,66 & 0,66 & 0,67 & 0,67 & 0,68 & 0,68 & 0,67 & 0,67 \\
\hline $\mathrm{Mg} \#$ & 0,34 & 0,34 & 0,33 & 0,35 & 0,32 & 0,33 & 0,34 & 0,34 & 0,35 & 0,33 & 0,33 & 0,33 & 0,33 & 0,34 & 0,34 \\
\hline $\mathrm{Fe} / \mathrm{Fe}+\mathrm{Mg}$ & 0,66 & 0,66 & 0,67 & 0,65 & 0,68 & 0,67 & 0,67 & 0,66 & 0,66 & 0,67 & 0,67 & 0,67 & 0,68 & 0,66 & 0,66 \\
\hline $\mathrm{Mg} / \mathrm{Mg}+\mathrm{Fe}$ & 0,34 & 0,34 & 0,33 & 0,35 & 0,32 & 0,33 & 0,34 & 0,34 & 0,35 & 0,33 & 0,33 & 0,33 & 0,33 & 0,34 & 0,34 \\
\hline $\mathrm{Fe}^{2} / \mathrm{Fe}^{2}+\mathrm{Fe}$ & 0,96 & 0,91 & 0,95 & 0,94 & 0,97 & 0,94 & 0,89 & 0,94 & 0,90 & 0,89 & 0,97 & 0,92 & 0,91 & 0,96 & 0,90 \\
\hline $\mathrm{Fe}^{3} / \mathrm{Fe}^{2}+\mathrm{Fe}$ & 0,04 & 0,09 & 0,05 & 0,06 & 0,03 & 0,06 & 0,11 & 0,06 & 0,10 & 0,11 & 0,03 & 0,08 & 0,09 & 0,04 & 0,10 \\
\hline $\mathrm{Fe}^{2} / \mathrm{Mg}+\mathrm{Fe}^{2}$ & 0,66 & 0,64 & 0,66 & 0,63 & 0,68 & 0,66 & 0,64 & 0,64 & 0,63 & 0,64 & 0,66 & 0,65 & 0,65 & 0,65 & 0,64 \\
\hline Flogopita & 58,23 & 48,23 & 49,63 & 50,50 & 53,33 & 48,62 & 43,34 & 50,00 & 47,88 & 44,51 & 58,58 & 53,84 & 48,08 & 51,80 & 45,43 \\
\hline Ti-Flogopita & 20,69 & 19,83 & 19,38 & 17,90 & 23,24 & 23,59 & 20,60 & 20,05 & 22,05 & 21,21 & 17,18 & 18,04 & 22,46 & 18,31 & 20,15 \\
\hline FerroEastonita & 5,97 & 15,26 & 8,26 & 9,78 & 4,46 & 8,78 & 17,74 & 10,29 & 16,64 & 17,41 & 5,16 & 13,50 & 14,84 & 5,83 & 16,77 \\
\hline Moscovita & 1,00 & 2,54 & 1,38 & 1,63 & 0,74 & 1,46 & 2,96 & 1,72 & 2,77 & 2,90 & 0,86 & 2,25 & 2,47 & 0,97 & 2,80 \\
\hline Eastonita & 13,92 & 11,36 & 17,34 & 17,02 & 17,39 & 15,45 & 11,01 & 14,87 & 9,62 & 10,47 & 17,05 & 10,10 & 10,43 & 18,69 & 10,33 \\
\hline Talco & 0,19 & 2,78 & 4,02 & 3,17 & 0,84 & 2,10 & 4,35 & 3,08 & 1,02 & 3,51 & 1,18 & 2,28 & 1,73 & 4,41 & 4,53 \\
\hline
\end{tabular}

Las formulas estructurales para las biotitas muestran que los cationes de $\mathrm{Si}\left(5,48-5,60\right.$ a.p.u.f) y $\mathrm{Al}^{\mathrm{IV}}(2,40-2,52$ a.p.u.f) llenan los sitios tetraédricos (TABLA 1). Por otro lado, los sitios octaédricos exhiben ligeramente más variabilidad entre 5,74 a 5,99 cationes a.p.u.f (promedio 5,89 a.p.u.f). Los otros 12 sitios de coordinación oscilan entre 1,87 a 1,99 cationes a.p.u.f (promedio de 1,93 a.p.u.f). Todo esto sugiriendo que las biotitas se encuentran cerca de los valores estequiométricos ideales (Yavuz et al., 2002).

El estudio petrográfico microtextural mostró que la biotita tiene un origen primario o magmático, lo cual se confirmó químicamente usando el diagrama ternario $10 \mathrm{TiO}_{2}-$ $\mathrm{FeO}^{\mathrm{T}}(=\mathrm{FeO}+\mathrm{MnO})-\mathrm{MgO}$ de Nachit et al. (2005), que discrimina la biotita magmática o primaria de la reequilibrada y secundaria. En este diagrama (FIGURA 4), las muestras de biotita en la SMRU se disponen en el campo de la "biotita primaria" con algunas muestras próximas del límite para el campo de las "biotitas primarias re-equilibradas", sin embargo, bien distantes de los valores de $\mathrm{TiO}_{2}$ de biotitas secundarias, es decir, demostrándose su origen magmático. Por otro lado, las biotitas analizadas muestran contenidos de $\mathrm{Al}$ octaédrico siempre menores que 1 (basado en 22 oxígenos; TABLA 1), como criterio de ser estrictamente magmáticas, ya que biotitas secundarias presentan mayor contenido de $\mathrm{Al}$ en este sitio (Nachit et al., 2005). No obstante, y aunque en la FIGURA 4 se muestra como biotitas primarias, en este trabajo fueron separadas estas biotitas en dos subgrupos composicionales denominados biotitas-IA (Bt-IA), caracterizadas por presentar petrográficamente menos deformación, y biotitas-IB (Bt-IB) con mayor evidencia de deformación. Las diferencias de composición entre los especímenes de biotita-IA y biotita-IB pueden distinguirse en varios puntos analizados. En todas las muestras de biotita, ya sean IA o IB hay suficiente Al para llenar completamente los sitios tetraédricos y hay un excedente de aluminio transportado a los sitios octaédricos, sin embargo, la diferencia de su contenido total de Al no hace una distinción entre la biotita IA e IB. El contenido total de $\mathrm{Al}$ de biotita IA está en el rango de 2,72 a 2,89 (a.p.u.f), mientras que en IB en el rango de 2,50 a 2,57 (a.p.u.f). 


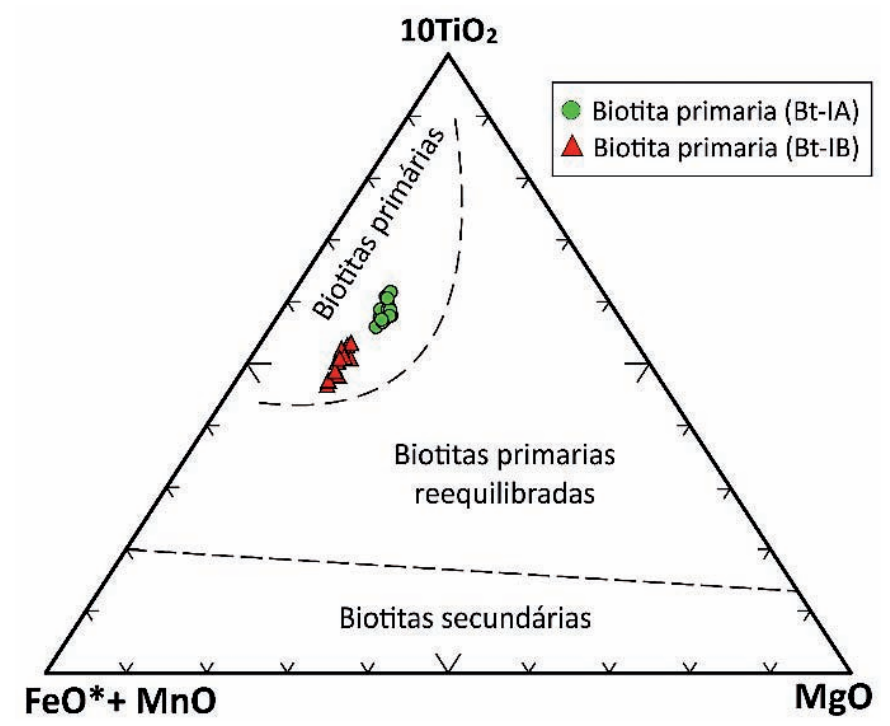

FIGURA 4. Composición química de biotitas de la SMRU en el diagrama ternario $10 \mathrm{TiO}_{2}-\mathrm{FeO}^{\mathrm{T}}(=\mathrm{FeO}+\mathrm{MnO})$ - $\mathrm{MgO}$ de $\mathrm{Nachit}$ et al. (2005) discriminando entre biotita magmática o primaria de la re-equilibrada y secundaria. En este diagrama fueron subclasificadas, en conjunto con los aspectos petrográficos, en biotitas sin mayor evidencia de deformación mineral (Bt-IA) y biotitas con evidencia de deformación (Bt-IB).

En el diagrama cuadrilátero (anita - siderofilita flogopita - eastonita) para clasificación de micas de Speer (1984), el cual muestra la relación composicional entre $\mathrm{Al}^{\mathrm{IV}}$ vs ( $\mathrm{Fe} / \mathrm{Fe}+\mathrm{Mg}$; FIGURA $\left.5 \mathrm{~A}\right)$, ambas biotitas muestran composiciones enriquecidas en la molécula de siderofilita, ocupando dos campos distintos y definidos por un rango relativamente grande de valores de $\mathrm{Fe} / \mathrm{Fe}+\mathrm{Mg}$, de $0,53-0,57$ para las biotitas IA y entre 0,65-0,68 para las biotitas IB. Los valores de aluminio tetraédrico $\left(\mathrm{Al}^{\mathrm{IV}}\right)$ presentan, en general para ambas biotitas, un rango pequeño con valores entre 2,40 a 2,52 a.p.u.f. En otras palabras, las biotitas estudiadas presentan una tendencia de incremento de la razón $\mathrm{Fe} /$ $\mathrm{Fe}+\mathrm{Mg}$ con valores constantes de $\mathrm{Al}$, y siendo este último menos discriminante para las rocas estudiadas.

Foster (1960) postuló un diagrama ternario relacionando el $\mathrm{Mg}-\left(\mathrm{Al}^{\mathrm{VI}}+\mathrm{Fe}^{3+}+\mathrm{Ti}\right)-\left(\mathrm{Fe}^{2+}+\mathrm{Mn}\right)$ para clasificar químicamente biotitas por su afinidad química, discriminándolas entre biotitas ricas en hierro, magnesio, flogopitas ricas en hierro y eastonitas. En este diagrama (FIGURA 5B), las biotitas pertenecientes a la SMRU fueron lanzadas en el campo de las "Mgbiotitas" para el grupo de biotitas IA y "Fe-biotitas" en el grupo de las biotitas IB. De manera similar, en la FIGURA 5C cuando comparados los contenidos de $\mathrm{MgO}$ con el $\mathrm{FeO}$ total, muestra que las biotitas menos deformadas (Bt-IA) presentan mayor contenido de $\mathrm{MgO}$ y valores $\mathrm{Fe} / \mathrm{Fe}+\mathrm{Mg}$ relativamente bajos en el intervalo de 0,53 a 0,57 , mientras que las biotitas con evidencias de deformación (Bt-IB) contienen más FeO y un $\mathrm{Mg} /(\mathrm{Mg}+\mathrm{Fe})$ relativamente menor entre 0,32 a 0,35 , es decir, que las composiciones de las biotitas en IA son ricas en $\mathrm{Mg}$, mientras que en las de IB se encuentran enriquecidas en Fe. Considerando lo anterior, Speer (1984) enfatiza que en la composición de la biotita se debe considerar que esta puede ser afectada, en mayor o menor grado, por procesos de reequilibrio tardío, tales como procesos de sustitución catiónicas o aniónicas, que son facilitadas por su estructura filosilicática. Siguiendo las posibles sustituciones propuestas por Stussi y Cuney (1996), las biotitas estudiadas presentan sustituciones de tipo $\mathrm{Fe}-\mathrm{Mg}_{-1}$, mostrando que en las biotitas con menos deformación para las de mayor evidencia de deformación se presenta una disminución de Mg en la estructura cristalina, mientras aumenta el Fe. Para Harrison (1990), la composición de la biotita puede ser reequilibrada cuando fluidos supersaturados ocurren, ya sean en rocas de composición granítica altamente fraccionados, durante las etapas de cristalización tardía o subsolidus. Este reequilibrio también puede ser atribuido a la localización de estas muestras en las proximidades de una zona de cizallamiento, la cual es una zona termal que posibilita la conducción de fluidos. Considerando esto, es evidenciado que en las biotitas el incremento de $\mathrm{Fe}$ con disminución de $\mathrm{Mg}$, explica e corrobora los dados petrográficos y microtexturales, que las rocas de la SMRU fueron afectados por procesos tectono-metamórficos, generando evidencias de comienzos de reequilibrio en las biotitas en zonas de cizallamiento. 
A)

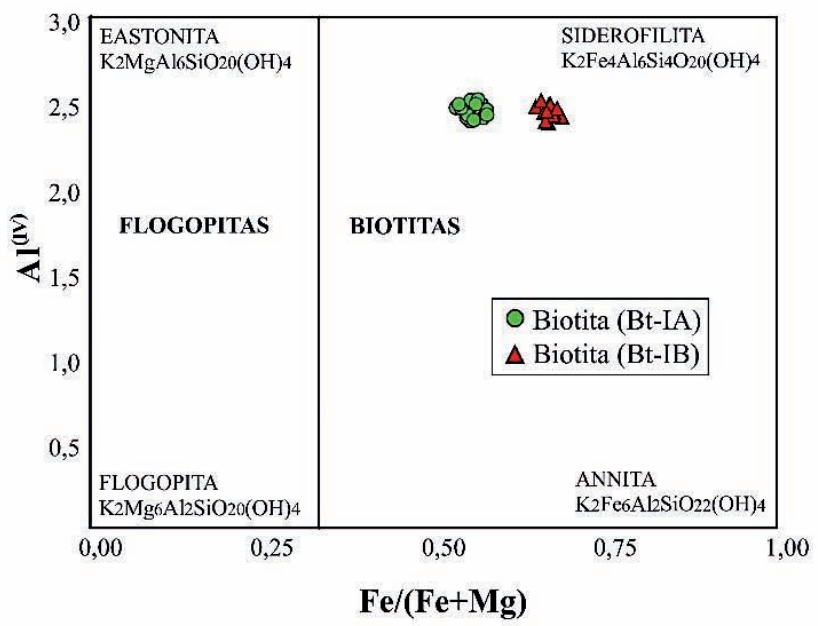

B)

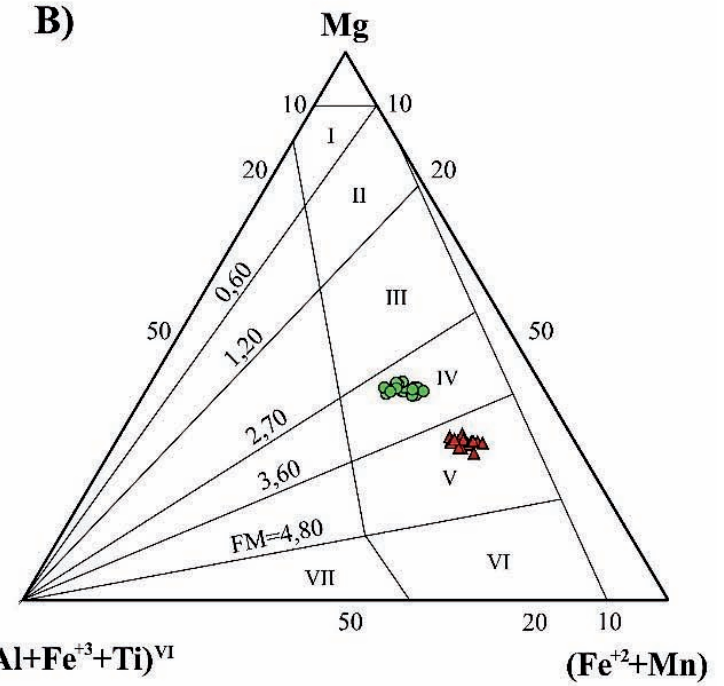

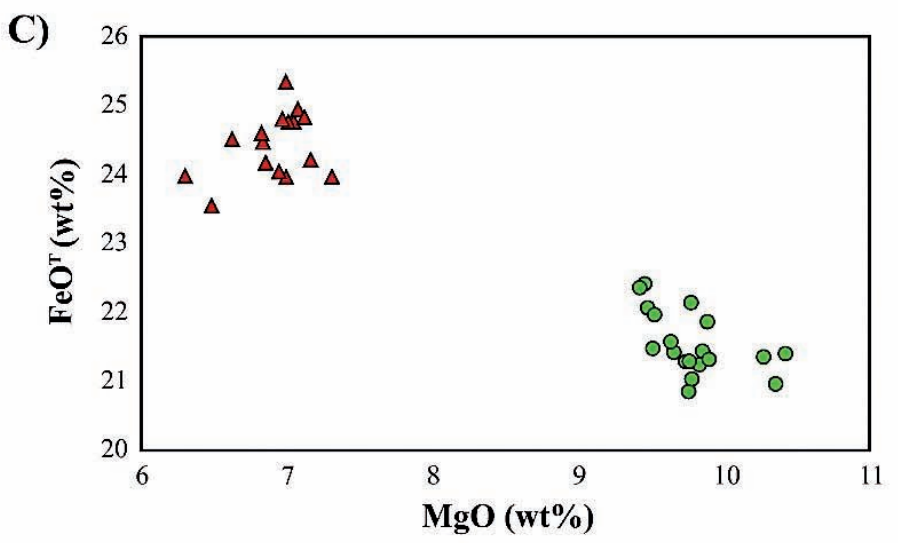

FIGURA 5. Diagramas de clasificación para las biotitas de la SMRU. A. Diagrama cuadrilátero clasificatorio $\mathrm{Al}{ }^{\mathrm{IV}} v s \mathrm{Fe} /(\mathrm{Fe}+\mathrm{Mg})$. B. Clasificación de biotitas por afinidad química $\mathrm{Mg}-\left(\mathrm{Al}^{\mathrm{VI}}+\mathrm{Fe}^{3+}+\mathrm{Ti}\right)-\left(\mathrm{Fe}^{2+}+\mathrm{Mn}\right)$, donde II = hierro-flogopita; III = eastonita; IV = Mg-biotita; V= Fe-biotita; adaptado de Foster (1960). C. Composición química de biotitas ploteadas en el diagrama MgO vs $\mathrm{FeO}^{\mathrm{T}}$. Nótese en todos los diagramas como las biotitas son separadas en dos grupos principales: biotitas ricas en $\mathrm{Mg}(\mathrm{Bt}-\mathrm{IA}) \mathrm{y}$ biotitas con enriquecimiento en $\mathrm{Mg}$ (Bt-IB).

\section{DETERMINACIÓN DE FUGACIDAD DE OXÍGENO Y CONDICIONES DE CRISTALIZACIÓN}

La biotita es un excelente indicador para el estado de oxidación del magma del cual esta fase mineral cristalizó (Wones y Eugster, 1965; Barriére y Cotton, 1979; Albuquerque, 1973; Noyes et al., 1983). El trabajo experimental de Wones y Eugster (1965) establece claramente la relación entre la composición de biotita y la fugacidad de oxígeno $\left(\mathrm{fO}_{2}\right)$, haciendo de este mineral un indicador valioso de las condiciones redox en magmas graníticos. Para efectos de obtener datos representativos y confiables respecto a la $\mathrm{fO}_{2}$, en la FIGURA 6 la composición de las biotitas IA, siendo las biotitas que representan las condiciones iniciales de cristalización de esta fase mineral, sin evidencia o mínimas transformaciones subsolidus en la SMRU, fueron ploteadas en el diagrama $\mathrm{Fe}^{3+}-\mathrm{Fe}^{+2}-\mathrm{Mg}$ de Wones y Eugster (1965), junto con los tres límites marcados de fugacidad de oxigeno: cuarzo-fayalita-magnetita (QFM), oxido de Niquel-Niquel (NNO) y hematitamagnetita (HM). En este diagrama (FIGURA 6), las biotitas estudiadas se grafican en el campo del NNO (Ni-NiO) con una leve tendencia para el campo del QFM $\left(\mathrm{SiO}_{2}-\mathrm{Fe}_{2} \mathrm{SiO}_{4}-\mathrm{Fe}_{3} \mathrm{O}_{4}\right)$. A partir de este diagrama, se puede observar que la relación $\mathrm{Fe}^{3+} /\left(\mathrm{Fe}^{+2}+\mathrm{Fe}^{3+}\right)$ es aproximadamente 0,30 para el límite del campo $\mathrm{Fe}_{3} \mathrm{O}_{4}$ $\mathrm{Fe}_{2} \mathrm{O}_{3},<0,25$ para el campo de $\mathrm{Ni}-\mathrm{NiO}$ (NNO), 0,10 para $\mathrm{SiO}_{2}-\mathrm{Fe}_{2} \mathrm{SiO}_{4}-\mathrm{Fe}_{3} \mathrm{O}_{4} ; \mathrm{y}<0,02$ para $\mathrm{Fe}_{1-\mathrm{x}} \mathrm{O}-\mathrm{Fe}_{3} \mathrm{O}_{4}$. Como 
las biotitas estudiadas muestran que el $\mathrm{Fe}^{3+} /\left(\mathrm{Fe}^{+2}+\mathrm{Fe}^{3+}\right)$ oscila entre 0,20 y 0,28 , estas claramente coinciden con el limite NNO.

Sin embargo, la aplicabilidad de estas proyecciones composicionales va a depender siempre del contenido de $\mathrm{Fe}^{3+}$, ofreciendo sólo una idea cualitativa de la fugacidad de oxígeno. Es posible obtener una estimación cuantitativa de la fugacidad de oxígeno o temperatura utilizando el trabajo experimental a partir de las curvas calibradas de Wones y Eugster (1965) mediante el diagrama $\mathrm{fO}_{2}-\mathrm{T}$ con 2070 bares de presión, esto si la biotita coexiste con magnetita + feldespato alcalino (Speer, 1984), lo cual es consistente con las paragénesis mostradas por BallesterosCamaro (2017) y otros autores para estas rocas, donde la presencia de biotita coexistiendo con feldespato alcalino y minerales de óxidos de $\mathrm{Fe}-\mathrm{Ti}$, proporcionan la base para estimar tentativa y cuantitativamente la fugacidad de oxígeno. Las rocas de composición granítica pertenecientes a la SMRU, equilibradas según los parámetros propuestos por Wones y Eugster (1965) $\mathrm{y}$ asumiendo un rango razonable de temperaturas de cristalización de 750 a $900^{\circ} \mathrm{C}$ para estas rocas, sugieren que estas biotitas fueron cristalizadas con una fugacidad de oxigeno entre $-12,71$ y $-13,66$, con condiciones en el límite de campo NNO (FIGURA 7).

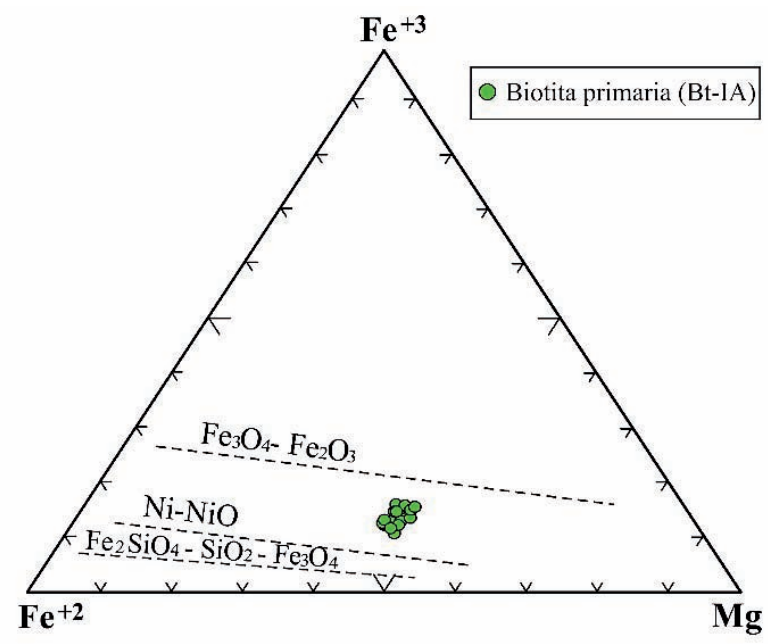

FIGURA6. Diagrama ternario $\mathrm{Fe}^{3+}-\mathrm{Fe}^{2+}-\mathrm{Mg}^{2+}$ de Wones y Eugster (1965) para la estimación de las condiciones redox de cristalización de biotitas estrictamente primarias (Bt-IA) de la SMRU, junto con los tres campos de fugacidad de oxigeno delimitados: cuarzofayalita-magnetita $\left(\mathrm{Fe}_{2} \mathrm{SiO}_{4}-\mathrm{SiO}_{2}-\mathrm{Fe}_{3} \mathrm{O}_{4}\right)$, oxido de Niquel-Niquel (Ni-NiO) y hematita-magnetita $\left(\mathrm{Fe}_{3} \mathrm{O}_{4}-\mathrm{Fe}_{2} \mathrm{O}_{3}\right)$.

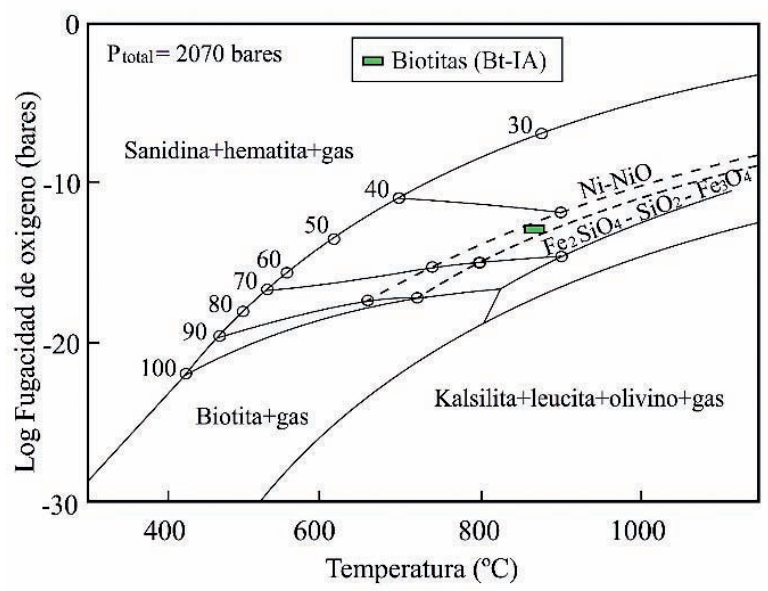

FIGURA 7. Diagrama de variación de condiciones Log $f\left(\mathrm{O}_{2}\right)$ - T de Wones y Eugster (1965), mostrando las condiciones de cristalización de las biotitas primarias en las rocas graníticas pertenecientes a la SMRU. Los valores de 0-100 representan la estabilidad de la biotita en función de $(100 \times \mathrm{Fe} /(\mathrm{Fe}+\mathrm{Mg}))$. 
Por otro lado, el geotermómetro propuesto por Henry et al. (2005), que evalúa el contenido de Ti en biotita también es utilizado para estimar las temperaturas de cristalización de las biotitas primarias de la SMRU, mediante la siguiente expresión $\mathrm{T}=([\ln (\mathrm{Ti})-a-$ $\left.\left.c\left(\mathrm{X}_{\mathrm{Mg}}\right)^{3}\right] / b\right)^{0,333}$, en donde $\mathrm{X}_{\mathrm{Mg}}=\mathrm{Mg} /(\mathrm{Mg}+\mathrm{Fe})$, Ti es el número de átomos por unidad de formula (a.p.u.f) basado en 22 oxígenos, $a=-2,359, b=4,6482 \times 10^{-9}$, y $c=$ $-1,728$. La precisión del geotermómetro Ti en biotita se estima en $\pm 24^{\circ} \mathrm{C}$ en el rango de temperatura más bajo y siendo mejorado a $\pm 12^{\circ} \mathrm{C}$ a temperaturas más altas, y con presión calibrada entre 4-6 kbar. De acuerdo a lo anterior, las temperaturas calculadas para estas biotitas de la SMRU oscilan entre 720 y $760^{\circ} \mathrm{C}$.

\section{CONSIDERACIONES PETROGENÉTICAS}

Mediante la química mineral en biotitas y la aplicación de varios modelos de diagramas de discriminación de AbdelRahman (1994), usando la composición de elementos principales de minerales de biotita en todo el espectro de rocas ígneas cristalizadas, es posible demostrar la naturaleza a partir de tres tipos distintos de magma de diversas fuentes y diferentes historias petrogenéticas. La composición química de las biotitas de la SMRU, en el diagrama de discriminación ternario $\mathrm{FeO}^{\mathrm{T}}-\mathrm{MgO}-\mathrm{Al}_{2} \mathrm{O}_{3} \mathrm{y}$ en los diagramas que correlacionan $\mathrm{Al}_{2} \mathrm{O}_{3} v s \mathrm{FeO}^{\mathrm{T}}, \mathrm{Al}_{2} \mathrm{O}_{3}$ vs $\mathrm{MgO}$ y $\mathrm{MgO}$ vs $\mathrm{FeO}^{\mathrm{T}}$ de Abdel-Rahman (1994), así como en el diagrama $\mathrm{Al}^{\mathrm{T}}$ vs $\mathrm{Mg}$ de Nachit et al. (1985), las muestras analizadas fueron ploteadas en el campo relativo a los granitos calco-alcalinos; en su mayoría granitos tipo I formados en ambientes orogénicos (FIGURA 8). Por otro lado y al graficarse conjuntamente los datos obtenidos en el diagrama cuadrilátero de Speer (1984), con los limites trazados en el trabajo de Jiang et al. (2002), distinguiendo entre biotitas formadas a partir de magmas tipo-I, tipo-A y shonshoniticos, las biotitas primarias analizadas coinciden con los diagramas de Abdel-Rahman (1994) y de Nachit et al. (1985), mostrándose en el campo de las biotitas asociadas a magmas tipo-I (FIGURA 9).
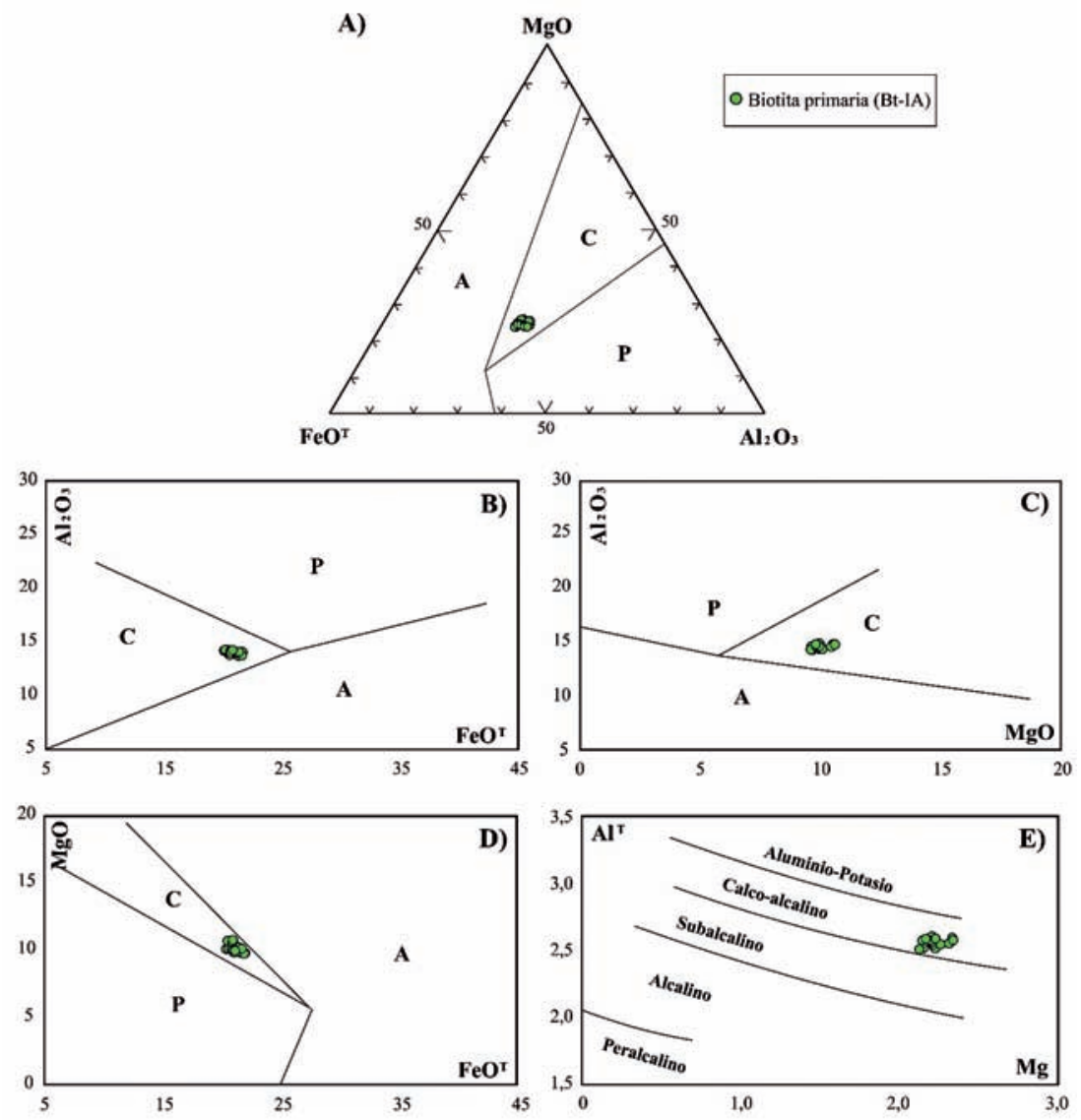

FIGURA 8. Diagramas discriminatorios para clasificación de series magmáticas a partir de la química de las biotitas, para las rocas de composición granítica de la SMRU. A-D. Diagramas de relación FeO - $\mathrm{MgO}-\mathrm{Al}_{2} \mathrm{O}_{3}, \mathrm{Al}_{2} \mathrm{O}_{3}$ vs $\mathrm{FeO}^{\mathrm{T}}, \mathrm{Al}_{2} \mathrm{O}_{3}$ vs $\mathrm{MgO}$ y $\mathrm{MgO}$ vs $\mathrm{FeO}^{\mathrm{T}}$ de Abdel-Rahman (1994). E. Diagrama $\mathrm{Al}^{\mathrm{T}}-\mathrm{Mg}$ para para clasificación de series magmáticas a partir de la química de las biotitas de Nachit et al. (1985). Simbología en diagramas: A= Serie alcalina asociada a granitos alcalinos anorogénicos; $\mathrm{P}=$ Granitos orogénicos peraluminosos y tipo-S; C=Serie Calco-alcalina asociada a granitos orogénicos tipo-I. 
Una observación que puede ser realizada en relación al diagrama $\mathrm{MgO}$ vs $\mathrm{FeO}^{\mathrm{T}}$ (Abdel-Rahman, 1994), es que el aumento de $\mathrm{Fe}$ y $\mathrm{Al}^{\mathrm{IV}}$ está acompañada de la disminución de $\mathrm{Mg}$, lo que podría estar relacionado, y como mencionado anteriormente, con un aumento de la fugacidad de oxigeno durante la evolución de las rocas que generaron esta suite.

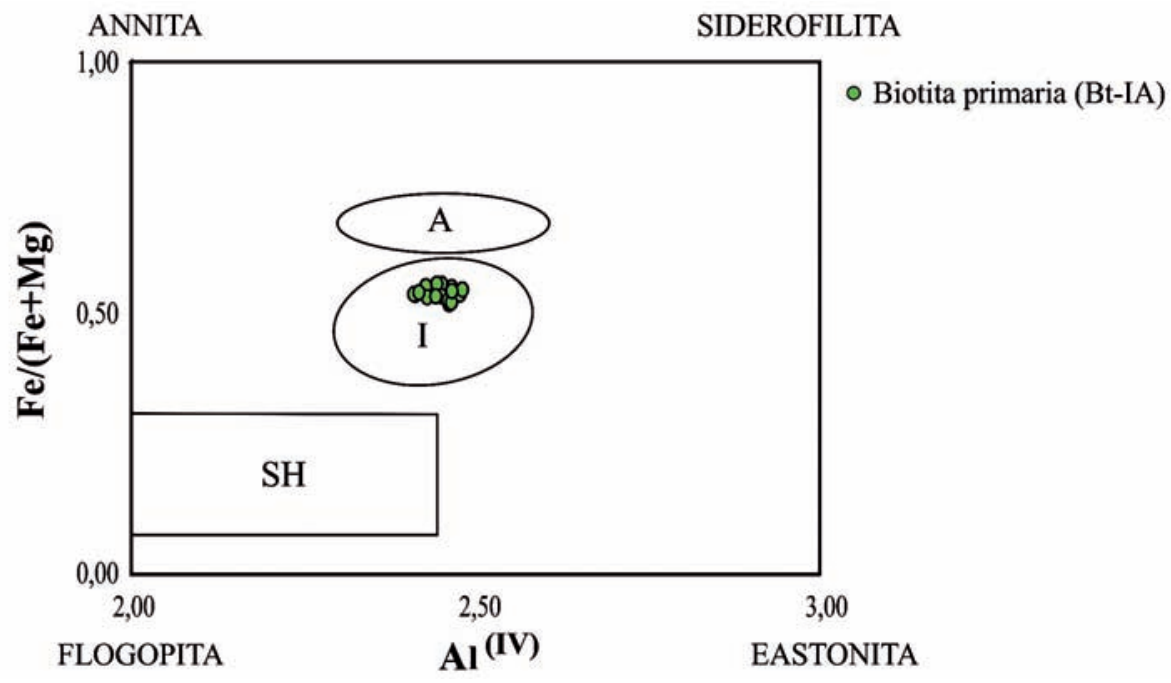

FIGURA 9. Diagrama $\mathrm{Fe}^{2+} /\left(\mathrm{Mg}^{2} \mathrm{Fe}^{2+}\right)$ vs $\mathrm{Al}^{\mathrm{IV}}$ adaptado por Jiang et al. (2002), para biotitas primarias de la SMRU, distinguiendo entre biotitas formadas a partir de magmas tipo-I, tipo-A y shonshoniticos. Simbología en diagrama: A= Granitos tipo-A; I= Granitos tipo-I; $\mathrm{SH}=$ plutones shonshoniticos.

\section{DISCUSIONES E INTERPRETACIONES}

Las microtexturas y la composición química de las biotitas estudiadas indican que esa fase mineral tiene un origen típicamente primario, aunque la presencia relativamente común de procesos tardíos como oxidación, cloritización y orientación en algunas secciones siguiendo una foliación metamórfica por deformación, muestra reequilibrio químico poscristalización, lo cual puede explicar el hecho de que en algunos secciones la biotita presente composición próxima en los límites de transición entre biotita primaria a primaria reequilibrada en el diagrama de Nachit et al. (2005). En general, las biotitas son minerales bastante sensibles a procesos posteriores que podrían haber afectado las rocas después de su solidificación, destacándose sobre este aspecto, principalmente, las biotitas encontradas en las secciones con mayor evidencia de deformación (BtIB), confirmándose variaciones en las condiciones, principalmente de temperatura, a partir del estudio de la composición química de las biotitas (Bt-IA) más próximas a la composición química global del protolíto ígneo de los neises de la SMRU. Estas variaciones son verificadas principalmente por sus diferencias químicas entre el $\mathrm{Fe}-\mathrm{Mg}$, en el cual las biotitas desde las menos deformadas a las más deformadas se destacan por su aumento significativo de Fe y disminución de $\mathrm{Mg}$, aproximándose levemente a composiciones flogopíticas. Este enriquecimiento en $\mathrm{FeO}^{\mathrm{T}}$ y empobrecimiento en $\mathrm{MgO}$ son interpretados como efectos de un metamorfismo regional progresivo asociados a zonas de cizallamiento característicos del Dominio Guiana Central relacionados a efectos tectónicos registrados para estas rocas durante el evento post-transamazónico.

En cuanto a la composición química de las biotitas, esta es generalmente utilizada para estimar las condiciones en la cual sus rocas magmáticas fueron generadas, siempre y cuando las biotitas estudiadas sean realmente magmáticas o primarias. Las biotitas estrictamente primarias estudiadas en la SMRU, muestran composiciones enriquecidas en la molécula de siderofilita, caracterizadas como Mg-biotitas trioctaédricas y presentando $\mathrm{Mg}^{\#}=0,43-0,47, \mathrm{Al}^{\mathrm{VI}}$ $=0,01$ a 0,08 a.p.u.f y $\mathrm{Fe}^{2+} /\left(\mathrm{Fe}^{2+}+\mathrm{Mg}\right)=0,45$ a 0,51 , con fracciones molares de $\mathrm{Mg}^{2+}, \mathrm{Fe}^{2+}$ y $\mathrm{Fe}^{3+}$ en la coordinación octaédrica, sugiriendo que su naturaleza es similar a biotitas primarias no afectadas por alteración hidrotermal (Beane, 1974). Estas biotitas, presentándose también con leve enriquecimiento en $\mathrm{Mg}$ y bajo $\mathrm{Al}^{\mathrm{VI}}$ reflejan un magma ligeramente fraccionado (Hecht, 1994), pudiendo ser un indicador 
relativo para el origen del magma parental (Burkhard, 1993; Lalonde y Bernard, 1993). Sin embargo, el enriquecimiento de $\mathrm{Fe}$ en minerales ferromagnesianos no es una consecuencia necesaria de la diferenciación de la cristalización, pero sí dependiendo en gran medida de la fugacidad de oxígeno y del agua (Mueller, 1972). En este sentido, la razón $\mathrm{Fe} /(\mathrm{Fe}+\mathrm{Mg})$ en biotitas son usadas como indicativas de las condiciones de fugacidad de oxigeno durante la cristalización (Wones y Eugster, 1965), donde en el caso específico de las biotitas estrictamente primarias estudiadas, esa relación es relativamente alta con valores en el intervalo de 0,53 a 0,57 , donde comparando estas razones con aquellas encontradas en los granitos estudiados por Lalonde y Bernard (1993), sugieren condiciones de fugacidad de oxígeno en el campo $\mathrm{NNO}$ (valores de $\mathrm{Fe} /(\mathrm{Fe}+\mathrm{Mg} \geq$ 0,50 ), y las cuales fueron verificadas en este trabajo mediante el diagrama ternario de Wones y Eugster (1965), sugiriendo así, condiciones más reductoras durante la cristalización con valores bajos de $\log \mathrm{O}_{2} \approx$ -12 a -13 . Este rango de valores indica que los magmas cristalizaron en condiciones de relativa baja fugacidad de oxígeno compatibles con la presencia de magnetita y con la composición rica en magnesio de las primarias (Bt-IA). Adicionalmente, estos resultados se encuentran consistentes con la presencia de la paragénesis mineral en equilibrio de titatina + magnetita + cuarzo en las rocas de composición granítica de los neises de la SMRU, donde de hecho, la presencia de Mg-biotita, magnetita, alanita y titanita en estas rocas, son una indicativa de la condición reductora del magma parental y reflejando condiciones relativamente bajas de fugacidad de oxigeno (Wones, 1989; Ishihara, 1997; Broska, 2003), siendo que tanto las relaciones de $\mathrm{Fe} /(\mathrm{Fe}+\mathrm{Mg})$ como la fugacidad de oxigeno indicadas, apuntan para una fuente típicamente de corteza para los magmas que dieron origen a esos granitos (Lalonde y Bernard, 1993). Para verificar lo anterior, Zhou (1986) sugirió que se podría usar un diagrama con la relación $\mathrm{FeO}$ / $(\mathrm{FeO}+\mathrm{MgO})$ frente a $\mathrm{MgO}$, usando composiciones de biotita para discriminar entre granitos de diferentes orígenes, ya sea de corteza $(\mathrm{C})$, mixta de manto-corteza (MC) y de manto (M). Utilizando dicho diagrama, las biotitas de la SMRU se posicionan dentro del área de granitos derivados de corteza (C), siendo verificadas con lo mencionado anteriormente (FIGURA 10).

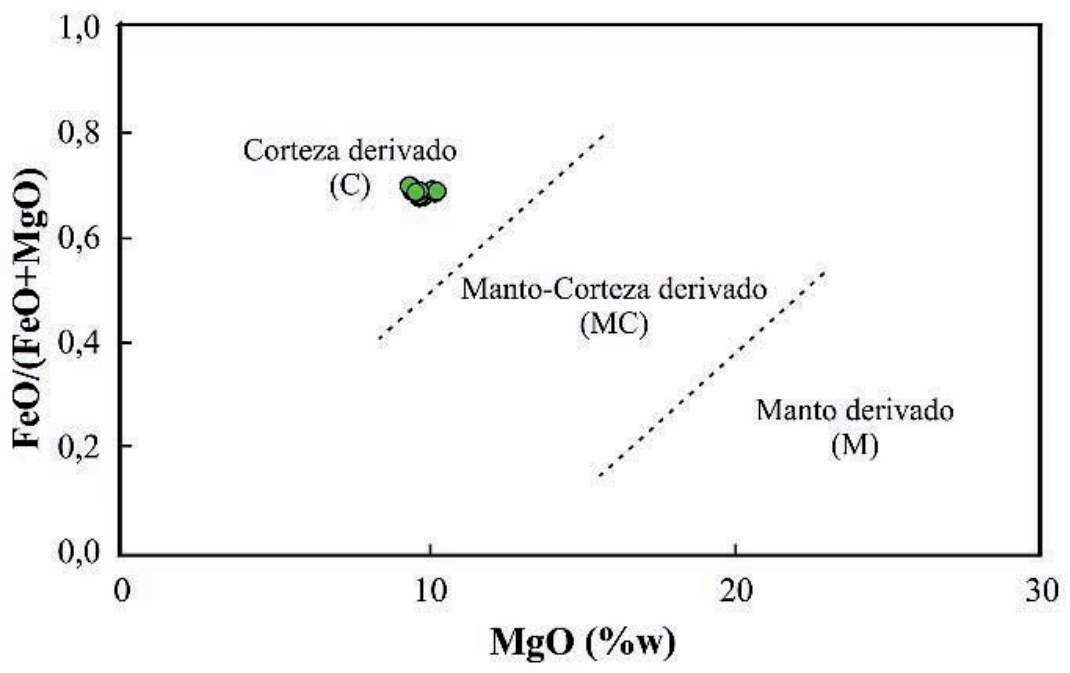

FIGURA 10. Diagrama de variación entre la relación $(\Sigma \mathrm{FeO}) /(\Sigma \mathrm{FeO}+\mathrm{MgO}) v s \mathrm{MgO}$, para discriminar entre granitos derivados de corteza (C), manto-corteza (MC) y de manto (M) a partir de la química de las biotitas, para las rocas de composición granítica de la SMRU, diagrama adaptado de Zhou (1986).

$\mathrm{Al}$ analizar en detalle las relaciones de Fe-Mg para las biotitas estrictamente primarias (Bt-IA), estas muestran un aumento relativo en $\mathrm{Mg}$ (1,08 a 1,19 a.p.u.f), en contraste con la disminución de $\mathrm{Fe}^{+3}$, sugiriendo un cambio muy pequeño en la fugacidad de oxígeno. Para Speer (1984) las biotitas presentan una tendencia al volverse significativamente más magnesianas cuando analizadas su índice de solidificación (IS), indicando una cristalización progresiva, siendo que para las biotitas estudiadas muestran un índice de solidificación entre 22,61 y 25,35, y cuando analizados en el diagrama de la relación $\mathrm{FeO} /(\mathrm{FeO}+\mathrm{MgO})$ con el IS de Speer (1984), muestran claramente para estas rocas una solidificación progresiva (FIGURA 11). 


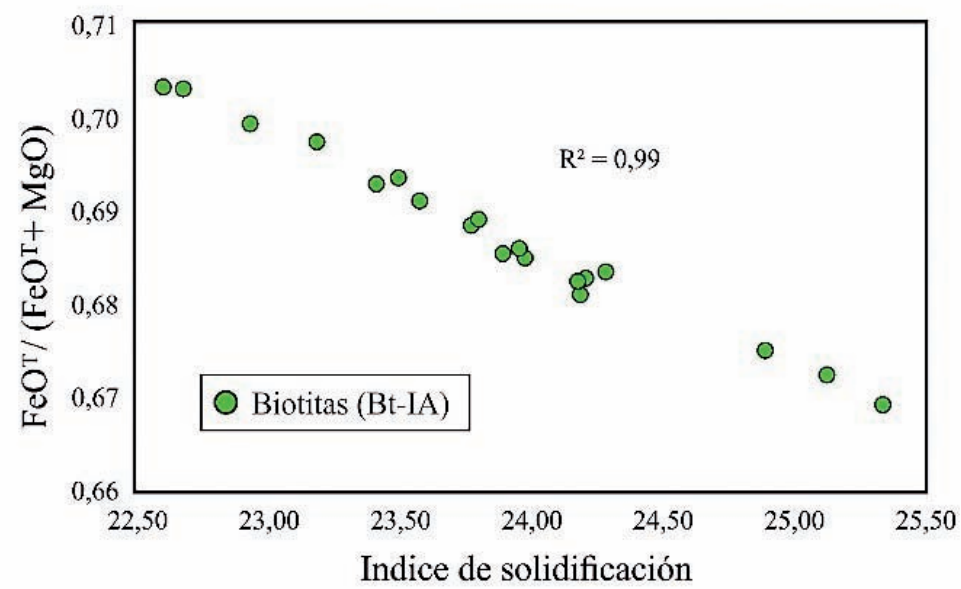

FIGURA 11. Diagrama de relación entre $\mathrm{FeO}^{\mathrm{T}} /\left(\mathrm{FeO}^{\mathrm{T}}+\mathrm{MgO}\right)$ de las biotitas primarias estudiadas de la SMRU y el índice de solidificación de la roca (IS). El IS es $100 * \mathrm{MgO} /\left(\mathrm{MgO}+\mathrm{FeO}^{\mathrm{T}}+\mathrm{Na}_{2} \mathrm{O}+\mathrm{K}_{2} \mathrm{O}\right)$, donde los óxidos están dados en porcentaje en peso (Speer, 1984).

Las temperaturas aquí determinadas mostraron que las rocas graníticas de la SMRU la temperatura de cristalización de las biotitas oscila entre 720 y $760^{\circ} \mathrm{C}$, indicando que estas biotitas cristalizaron a altas temperaturas (Deer et al., 1966). Para Dodge y Moore (1968), las biotitas en rocas graníticas a dioríticas generalmente cristalizan en un intervalo amplio de temperaturas entre 700 y $900^{\circ} \mathrm{C}$, y mencionan que las biotitas magnesianas son más estables y pueden contener más halógenos que las biotitas ricas en Fe a alta temperatura (Mueller, 1972), por lo que en ese caso, la química de la biotita puede producir composiciones más fluidas, lo que puede acelerar el aumento de la temperatura (Speer, 1984), siendo que los datos obtenidos en este trabajo por el método del contenido de Ti en biotita, pueden considerarse como coherentes para esta fase mineral.

La composición química de la biotita también se caracteriza por ser un indicador altamente confiable en cuanto al contexto tectónico de las rocas en las cuales esas biotitas fueron formadas. Abdel-Rahman (1994) en su trabajo definió: 1) biotita típica de granitos orogénicos tipo-I (de afinidad calco alcalinas-C); 2) biotita de granitos anorogénicos alcalinos (de afinidad alcalinos-A); y 3) biotita de granitos peraluminosos, incluyendo los típicos tipo-S (de afinidad peraluminoso-P). La biotita de los neises de composición granítica de la SMRU estudiados muestra en general geoquímica semejante a biotita de rocas pertenecientes a granitos orogénicos calco-alcalinos tipo-I. En ese mismo trabajo, Abdel-Rahman (1994) encontró que las biotitas que están presentes en los granitos de tipo I son relativamente enriquecidas en magnesio (Shabani et al., 2003; Lalonde y Bernard, 1993), mientras que las biotitas de granito de tipo $\mathrm{S}$ están relativamente enriquecidas en aluminio. Con todo esto, y sabiendo que las biotitas primarias estudiadas son magnesianas, soportaría la clasificación de rocas generadas a partir de magmas tipo I, los cuales son magmas característicos de ambientes de subducción (Chappell y White, 1974; White, 1979; Petro et al., 1979; White y Chappell, 1983; Barbarin, 1990, 1999; Altherr et al., 2000). Por otro lado, para Roberts y Clemens (1993), magmas calco-alcalinos pueden ser generados a partir de la fusión parcial de rocas metaígneas máficas a intermedias de naturaleza calco-alcalina hasta, posiblemente, calco-alcalina de alto-K existentes en la corteza inferior, por lo que para el Dominio Guiana Central (DGC), la fuente de estas rocas calco-alcalinas asociadas a la SMRU, podrían estar asociados al manto litosférico subcontinental enriquecido durante la orogénesis Transamazónica.

Finalmente, y al comparar los datos obtenidos para biotitas en rocas neísicas aflorantes en la Serra Repartimento con los datos geoquímicos en roca total presentados para los neises biotítico-hornbléndicos de composición mozogranítica de la SMRU en el trabajo de Fraga (2000) dentro del proyecto Roraima Central, llama la atención el hecho de encontrase altamente coherentes, mostrando compatibilidad con granitóides calco-alcalinos asociados a magmas tipo I. 


\section{CONCLUSIONES}

Los estudios petrográficos y de análisis de química mineral en biotitas de la SMRU nos permitieron llegar a las siguientes conclusiones.

- Las biotitas primarias dentro de los neises de la SMRU, caracterizadas por presentar menor deformación por efectos de metamorfismo, y en composición química más próximas a la del protolíto ígneo son ricas en $\mathrm{Mg}$ y pobres en $\mathrm{Fe}$, clasificadas como magnesio-biotitas enriquecidas en la molécula de siderofilita.

- Las temperaturas de cristalización de las biotitas de la SMRU oscilaron entre 720 y $760^{\circ} \mathrm{C}$, y con base en sus composiciones químicas, se puede inferir que los granitos estudiados cristalizaron bajo condiciones de relativa baja fugacidad de oxígeno, en el límite de campo NNO, lo que es coherente con la presencia en estos granitos de la paragénesis primaria titanita + magnetita +cuarzo.

- La composición de la biotita muestra claramente una correlación directa con la geoquímica de sus granitoides hospederos, donde para las rocas de composición granítica de la SMRU, se muestran típicamente orogénicos tipo-I y de afinidad con asociaciones calco-alcalinos relativamente enriquecidas en magnesio, lo cuales se originaron a partir de una fuente de corteza.

\section{AGRADECIMIENTOS}

Este trabajo está basado, en parte, en la disertación de maestría desarrollada por el autor principal en el Programa de Postgrado en Geociencias de la Universidad Federal do Amazonas (UFAM). Los autores agradecen a la CAPES por el apoyo en la realización de maestría y desarrollo del proyecto "Geologia e gênese das ocurrências de Ti, Nb e ETR's na Serra Repartimento, $R R$ ” y a la Profa. Dra. Martha Edith Velasquez David por el seguimiento, apoyo en el proyecto y los aportes parciales del presente artículo. También agradecen al Laboratorio de Microssonda Electrónica de Universidad de Brasilia (UnB) por permitir realizar los análisis de química mineral, y a la Universidade Federal do Amazonas (UFAM) por el uso de los laboratorios de microscopia petrográfica y técnicas mineralógicas. Especial agradecimiento a los revisores externos por sus observaciones y comentarios detallados y constructivos.

\section{REFERENCIAS}

Abdel-Rahman, A.M. (1994). Nature of biotites from alkaline, calc-alkaline, and peraluminous magmas. Journal of Petrology, 35(2), 525-541. doi: 10.1093/ petrology/35.2.525.

Albuquerque, C.A.R. (1973). Geochemistry of biotites from granitic rocks, Northern Portugal. Geochimica et Cosmochimica Acta, 37(7), 17791802. doi: 10.1016/0016-7037(73)90163-4.

Almeida, F.F.M. de. (1978). A evolução dos crátons Amazônico e do São Francisco, comparada com a de seus homólogos do Hemisfério Norte. Congresso Brasileiro de Geologia, Vol. 6, Recife, Brasil.

Almeida, M.E. (2006). Evolução geológica da porção centro-sul do Escudo das Guianas com base no estudo geoquímico, geocronológico e isotópico dos granitóides paleoproterozóicos do sudeste de Roraima, Brasil. Tese de Doutorado. Centro de Geociências, Universidade Federal do Pará, Belém, Brasil.

Almeida, M.E., Ferreira, A.L, and Pinheiro, S.S. (2003). Associações graníticas do oeste do estado de Roraima, Domínio Parima, Escudo das Guianas, Brasil. Géologie de la France, 2-3-4, 134-159.

Altherr, R., Holl, A., Hegner, E., Langer, C., and Kreuzer, H. (2000). High-potassium, calc-alkaline I-type plutonism in the European Variscides: northern Vosges (France) and northern Schwartzwald (Germany). Lithos, 50(1-3), 51-73. doi: 10.1016/ S0024-4937(99)00052-3.

Ballesteros-Camaro, C.A. (2017). Geologia e gênese das ocorrências de Ti, $\mathrm{Nb}$ e ETR's na Serra Repartimento, Roraima. Tese de Mestrado, Universidade Federal do Amazonas, Brasil.

Barbarin, B. (1990). Granitoids: main petrogenetic classifications in relation to origin and tectonic setting. Geological Journal, 25(3-4), 227-238. doi: 10.1002/gj.3350250306. 
Barbarin, B. (1999). A review of the relationships between granitoid types, their origins and their geodynamic environments. Lithos, 46(3), 605-626. doi: 10.1016/S0024-4937(98)00085-1.

Barriére, M., and Cotton, J. (1979). Biotites and associated minerals as markers of magmatic fractionation and deuteric equilibration in granites. Contributions to Mineralogy and Petrology, 70(2), 183-192. doi: 10.1007/BF00374447.

Beane, R.E. (1974). Biotite stability in the porphyry copper environment. Economic Geology, 69(2), 241-256.

Broska, I. (2003). REE accessory minerals in the felsic silicic rocks of the west-carpathians: their distribution, composition and stability. Acta mineralogica-petrographica, Abstract Series 1, 15.

Burkhard, D.J.M. (1993). Biotite crystallization temperatures and redox states in granitic rocks as indicator for tectonic setting. Geologie en Mijnbouw, 71(4), 337-349.

Chappell, B.W., and White, A.J.R. (1974). Two contrasting granite types. Pacific Geology, 8, 173174.

Costa, U.A.P, Dantona, R.J.G., Neves, M.P., Splendor, F., Da Silva, M.B., and Abram, M.S. (2011). Petrografia de rochas anortosíticas do município de Iracema, Estado de Roraima. SBG, $12^{\circ}$ Simpósio de Geologia da Amazônia. Boa Vista, Roraima, Brasil. Anais.

CPRM. (1999). Roraima Central, Folhas NA.20-X-B e NA.20-X-D (integrais) e folhas NA.20-XA, NA.20-X-C, NA.21-V-A e NA.21-V-C (parciais). Escala 1:500.000. Brasília. Serviço Geológico do Brasil, 166 p. CD-ROM.

Deer, W.A., Howie, R.A., and Zussman, J. (1966). An introduction to the rock-forming minerals. London: Longman.

Deer, W.A., Howie, R.A., and Zussman, J. (1997). RockForming Minerals: Single-chain silicates. London: Longman.

Dodge, F.C.W., and Moore, J.G. (1968). Occurrence and composition of biotites from the cartridge pass pluton of the Sierra Nevada Batholith, California.
US Geological. Survey Profesional Paper, 600(B), 6-10.

Dymek, R.F. (1983). Titanium, aluminium and interlayer cation substitutions in biotite from high-grade gneisses, West Greenland. American Mineralogist, 68(9-10), 880-899.

Figueiredo, R.F. (2016). Contexto tectônico do complexo alcalino Apiaú-Roraima: aerogeofísica, petrologia e geocronologia U-Pb. Tese de Mestrado, Instituto de Geociências, Universidade Estadual de Campinas, Brasil.

Figueiredo, R.F., and Santos, T.J.S. (2015). Integração de dados aerogeofísicos e geológicos do Complexo Alcalino Apiaú, próximo a região de Campos Novos - Roraima. SBG, $14^{\circ}$ Simpósio de Geologia da Amazônia. Marabá, Anais.

Foster, M.D. (1960). Interpretation of the Composition of Trioctahedral Micas. U.S Geological Survey Professional Paper, 354(B): 1-49.

Fraga, L.M.B. (1999). Geologia Estrutural. In: Programa de Levantamento Geológicos Básicos do Brasil. Roraima Central, Folhas NA.20-X-B e NA.20-X-D (inteiras), NA.20-X-A, NA.20-X-C, NA.21-V-A e NA.21-V-C (parciais). Escala 1:500.000. Estado do Amazonas, Brasília: CPRM, 1999. Relatório final, cap. 4, p. 117-128.

Fraga, L.M.B. (2000). Suíte Metamórfica de Rio Urubu. In: CPRM, Programa Levantamentos Geológicos Básicos - PLGB. Roraima Central, Folhas NA.20-X e NA.21-V, Estado de Roraima, escala 1:500.000. Brasília/SUREGMA, pp. 127-177. 1 CD-ROM.

Fraga, L.M.B. (2002). A associação AnortositoMangerito Granito Rapakivi (AMG) do Cinturão Guiana Central, Roraima, e suas encaixantes paleoproterozóicas: Evolução Estrutural, Geocronologia e Petrologia. Tese de doutorado, Universidade Federal do Pará, Brazil.

Fraga, L.M.B., Almeida, M.E., and Macambira, M.J.B. (1997a). First lead-lead zircon ages of charnockitic rocks from Central Guiana Belt (CGB) in the state of Roraima, Brazil. $1^{\text {st }}$ South American Symposium on Isotope Geology, Campos do Jordão, São Paulo, Brasil. 
Fraga, L.M.B., Araújo, R.V. de., and Duarte, B.P. (1997b). Igneous charnockitic rocks of the Kanuku Complex and Serra da Prata Suite in the Central Guiana Belt (CGB), Roraima State, Brazil. International Symposium on Granites and Associated Rocks, Salvador.

Fraga, L.M.B., Dall'agnol, R., Costa, J.B.S., and Macambira, M.J.B. (2009a). The mesoproterozoic mucajaí anorthosite-mangerite-rapakivi granite complex, Amazonian craton, Brazil. The Canadian Mineralogist, 47(6), 1469-1492.

Fraga, L.M.B., Macambira, M.J.B., Dall'agnol, R., and Costa, J.B.S. (2009b). 1.94 - 1.93 Ga charnockitic magmatism from the central part of the Guyana Shield, Roraima, Brazil: Single-zircon evaporation data and tectonic implications. Journal of South American Earth Sciences, 27(4), 247-257. doi: 10.1016/j.jsames.2009.02.007.

Fraga, L.M.B., Nunes, N.S.V., and Riker S.R.L. (1994). Contribuição à Geologia da região do Rio Urubu: Um segmento do Cinturão de Cisalhamento Guiana Central. Congresso Brasileiro de Geologia, Vol. 38, Anais 2, Camboriú, Brasil.

Gaudette, H.E., Olszewski, W.J., and Santos, J.O.S. (1996). Geochronology of Precambrian rocks from the northern part of Guiana Shield, State of Roraima, Brazil. Journal of South American Earth Sciences, 9(3-4), 183-195. doi: 10.1016/08959811(96)00005-3.

Harrison, T.N. (1990). Chemical variation in micas from the Cairngorm pluton, Scotland. Mineralogical Magazine, 54(376), 355-366.

Hecht, L. (1994). The Chemical composition of biotite as an indicator of magmatic fractionation and metasomatism in Sn-specialised granites of the Fichtelgebirge (NW Bohemian Massif, Germany). In: R. Seltmann, H. Kämpf, P. Möller. Metallogeny of collisional orogens (pp. 295-300). Prague: Czech Geological Survey.

Heinonen, A., Fraga, L.M., Rämö, T., Dall’Agnol, R., Mänttäri, I., and Andersen, T. (2012). Petrogenesis of the igneous Mucajaí AMG complex, northern Amazonian craton - geochemical, $\mathrm{U}-\mathrm{Pb}$ geochronological, and $\mathrm{Nd}-\mathrm{Hf}-\mathrm{O}$ isotopic constraints. Lithos, 151, 17-34. doi: 10.1016/j. lithos.2011.07.016.
Henry, D.J., Guidotti, C.V., and Thomson, J.A. (2005). The Ti-saturation surface for low-to-medium pressure metapelitic biotites: Implications for geothermometry and Ti-substitution mechanisms. American Mineralogist, 90(2-3), 316-328. doi: 10.2138/am.2005.1498.

Ishihara, S. (1977). The magnetite-series and ilmeniteseries granitic rocks. Mining Geology, 27, 293-305.

Jacobs, D.C., and Parry, W.T. (1979). Geochemistry of biotite in the Santa Rita porphyry copper deposit, New Mexico. Economic Geology, 74(4), 860-887.

Jiang, Y., Jiang, S., Ling, H., Zhou, X., Rui, X., and Yang, W. (2002). Petrology and geochemistry of shoshonitic plutons from the western Kunlun orogenic belt, Xinjiang, northwestern China: implications for granitoid geneses. Lithos, 63(3-4), 165-187.

Kretz, R. (1983). Symbols of rock-forming minerals. American Mineralogist, 68(1-2), 277-279.

Lalonde, A.E., and Bernard, P. (1993). Composition and color of biotite from granites: two useful properties in the characterization of plutonic suites from the Hepburn internal zone of Wopmay orogen, Northwest Territories. Canadian Mineralogist, 31(1), 203-217.

Masoudi, F., and Jamshidi-Badr, M. (2008). Biotite and hornblende composition used to investigate the nature and thermobarometry of Pichagchi pluton, northwest Sanandaj-Sirjan metamorphic Belt, Iran. Journal of Sciences, Islamic Republic of Iran, 19(4), 329-338.

Mueller, R.F. (1972). Stability of biotite: A discussion. American Mineralogist, 57(1-2), 300-316.

Nachit, H., Ibhi, A., Abia, E.H., and Ohoud, M.B. (2005). Discrimination between primary magmatic biotites, reequilibrated and neoformed biotites. Comptes Rendus Geoscience, 337(16), 1415-1420. doi: 10.1016/j.crte.2005.09.002.

Nachit, H., Razafimahefa, N., Stussi, J.M., and Carron, J.P. (1985). Composition chimique des biotites et typologie magmatique des granitoides. Comptes Rendus de l'Académie des Sciences, 301(11), 813818. 
Noyes, H.J., Wones, D.R., and Frey, F.A. (1983). A tale of two plutons: petrographic and mineralogic constraints on the petrogenesis of the Red Lake and Eagle Peak plutons, Central Sierra Nevada, California. The Journal of Geology, 91(4), 353-378.

Petro, W.L., Vogel, T.A., and Wilband, J.T. (1979). Major-element chemistry of plutonic rock suites from compressional and extensional plate boundaries. Chemical Geology, 26(3-4), 217- 235.

Reis, N.J., Faria, M.S.G., Almeida, M.E., and Oliveira, M.A. (2004). Folhas NA.20-Boa Vista e NB.20Roraima. In: C. Schobbenhaus, J.H. Gonçalves, J.O.S. Santos, M.B. Abram, R. Leão Neto, G.M.M. Matos, R.M. Vidotti, M.A.B., Ramos, J.D.A. Jesus (eds.). Carta geológica do Brasil ao milionésimo: Sistema de Informações Geográficas - SIG. Brasilia: Programa Geologia do Brasil. CPRM, CD-ROM.

Roberts, M.P., and Clemens, J.D. (1993). Origin of high-potassium, calc-alkaline, I-type granitoids. Geology, 21(9), 825-828. doi: 10.1130/0091-7613(1993)021<0825:OOHPTA $>2$. 3.CO;2.

Rosa-Costa, L.T. (2006). Geocronologia ${ }^{207} \mathrm{~Pb} / 206 \mathrm{~Pb}$, $\mathrm{Sm}-\mathrm{Nd}$, U-Th- $\mathrm{Pb}$ E ${ }^{40} \mathrm{Ar}^{-39} \mathrm{Ar}$ do segmento Sudeste do escudo das Guianas: Evolução crustal e termocronologia do evento transamazônico. Tese de Doutoramento, Geoquímica e Petrologia, Universidade Federal do Pará, Belém, Brasil.

Santos, J.O.S., Hartmann, L.A., Gaudette, H.E., Groves, D.I., Mcnaughton, N.J., and Fletcher, I.R. (2000). A new understanding of the provinces of the Amazon Craton based on integration of field mapping and $\mathrm{U}-\mathrm{Pb}$ and $\mathrm{Sm}-\mathrm{Nd}$ geochronology. Gondwana Research, 3(4), 453-488. doi: 10.1016/ S1342-937X(05)70755-3.

Shabani, A.A.T., Lalonde, A.E., and Whalen, J.B. (2003). Composition of biotite from granitic rocks of the Canadian Appalachian Orogen: A potential tectonomagmatic indicator?. The Canadian Mineralogist, 41(6), 1381-1396.

Solie, D.N., and Su, S.C. (1987). An occurrence of Ba-rich micas from the Alaska Range. American Mineralogist, 72(9-10), 995-999.
Speer, J.A. (1984). Micas in igneous rocks. In: S.W. Bailey (ed.). Micas (pp. 299-356). Mineralogical Society of America, Reviews in Mineralogy and Geochemistry, Madison, Wisconsin, USA.

Speer, J.A. (1987). Evolution of magmatic AFM mineral assemblages in granitoid rocks: The hornblende + melt $=$ biotite reaction in the Liberty Hill pluton, South Carolina. American Mineralogist, 72(9-10), 863-878.

Stussi, J.M., and Cuney, M. (1996). Nature of biotites from alkaline, calc-alkaline and peraluminuos magmas by Abdel-Fattah M. Abdel-Haman: A comment. Journal of Petrology, 37(5), 1025-1029.

Tarazona, C.A. (2015). Análise das petrotramas das rochas charnockíticas da Serra da Prata, Mucajaí/ RR. Tese de Mestrado, Instituto de Ciências Exatas, Universidade Federal do Amazonas, Brasil.

Tassinari, C.C.G., Bettencourt, J.S., Geraldes, M.C., Macambira, M.J.B., and Lafon, J.M. (2000). The Amazonian craton. In: U.G. Cordani, E.J. Milani, A. Thomaz Filho, D.A. Campos (eds.). Tectonic evolution of South America (pp. 41-96). Rio de Janeiro.

Tassinari, C.C.G., and Macambira, M.J.B. (2004). A evolução tectônica do Cráton Amazônico. In: V. Mantesso-Neto, A. Bartorelli, C. Dal Ré Carneiro, B.B. Brito-Neves (eds.). Geologia do Continente Sul-Americano: Evolução da obra de Fernando Flávio Marques de Almeida (pp. pp. 471-485). São Paulo: Beca.

White, A.J.R. (1979). Sources of granite magmas. Geological Society of America, Abstracts with Programs, 11(7), 539.

White, A.J.R., and Chappell, B.W. (1983). Granitoid types and their distribution in the Lachlan Fold Belt, southeastern Australia. In: J.A. Roddick (ed.). Circum-Pacific Plutonic Terranes (pp. 21-34). Vol. 159. Boulder: Geological Society of America.

Wones, D.R. (1989). Significance of the assemblage titanite + magnetite + quartz in granitic rocks. American Mineralogist, 74(7-8), 744-749. 
Wones, D.R., and Eugster, H.P. (1965). Stability of biotite: experiment, theory, and application. American Mineralogist, 50(9), 1228-1272.

Yavuz, F. (2003). Evaluating micas in petrologic and metallogenic aspect: I-definitions and structure of the computer program MICA ${ }^{+}$. Computer and Geosciences, 29(10), 1203-1213. doi: 10.1016/ S0098-3004(03)00142-0.

Yavuz, F., Gültekin, A.H., Örgün, Y., Çelik, N., Karakaya, M.Ç., and Sasmaz, A. (2002). Mineral chemistry of barium- and titanium-bearing biotites in calc-alkaline volcanic rocks from the Mezitler area (Balıkesir Dursunbey), Western Turkey. Geochemical Journal, 36, 563-580.
Zhou, Z.X. (1986). The origin of intrusive mass in Fengshandong, Hubei province. Acta Petrologica Sinica, 2(2), 59-70.

\begin{tabular}{c}
\hline \hline Carlos Andrés Ballesteros-Camaro \\
ORCID: 0000-0002-4214-6417 \\
Johanna Ríos-Guerrero \\
ORCID: 0000-0003-4552-4855 \\
\hline \hline
\end{tabular}

Trabajo recibido: enero 31 de 2018

Trabajo aceptado: agosto 22 de 2018 